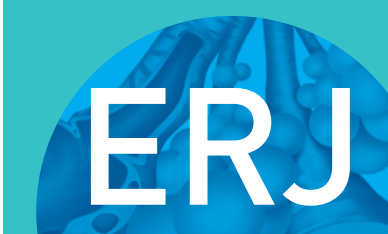

open research

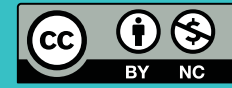

\section{Pulmonary function tests in type 2 diabetes: a meta-analysis}

\author{
Jesús Díez-Manglano (10 ${ }^{1}$ and Uxua Asin Samper ${ }^{2}$
}

Affiliations: ${ }^{1}$ Dept of Internal Medicine, Hospital Royo Villanova, Zaragoza, Spain. ${ }^{2}$ Dept of Internal Medicine, University Hospital Miguel Servet, Zaragoza, Spain.

Correspondence: Jesús Díez-Manglano, Duquesa Villahermosa 163, $8^{\circ}$ D, 50009 Zaragoza, Spain.

E-mail: jdiezQaaragon.es

\section{ABSTRACT}

Objectives: The aim of this study was to determine the association between type 2 diabetes (T2D) and pulmonary function tests.

Methods: After conducting an exhaustive literature search, we performed a meta-analysis. We employed the inverse variance method with a random-effects model to calculate the effect estimate as the mean difference (MD) and 95\% confidence interval (CI). We calculated the heterogeneity with the $\mathrm{I}^{2}$ statistic and performed a meta-regression analysis by sex, body mass index (BMI), smoking and geographical region. We also conducted a sensitivity analysis according to the studies' publication date, size of the T2D group and the study quality, excluding the study with the greatest weight in the effect.

Results: The meta-analysis included 66 studies (one longitudinal, two case-control and 63 cross-sectional), with 11134 patients with T2D and 48377 control participants. The pooled MD (95\% CI) for the predicted percentage of forced expiratory volume in $1 \mathrm{~s}\left(\mathrm{FEV}_{1}\right)$, forced vital capacity $(\mathrm{FVC})$, forced expiratory flow at $25-75 \%$ of FVC, peak expiratory flow, and diffusing capacity of the lung for carbon monoxide were -7.15 (95\% CI -8.27, -6.03; p<0.001), -9.21 (95\% CI -11.15, -7.26; $\mathrm{p}<0.001),-9.89$ (95\% CI $-14.42,-5.36$; $\mathrm{p}<0.001),-9.79(95 \% \mathrm{CI}-13.42,-6.15 ; \mathrm{p}<0.001)$ and $-7.13(95 \% \mathrm{CI}-10.62,-3.64 ; \mathrm{p}<0.001)$, respectively. There was no difference in the ratio of $\mathrm{FEV}_{1} / \mathrm{FVC}(95 \% \mathrm{CI}-0.27 ;-1.63,1.08 ; \mathrm{p}=0.69)$. In all cases, there was considerable heterogeneity. The meta-regression analysis showed that between studies heterogeneity was not explained by patient sex, BMI, smoking or geographical region. The findings were consistent in the sensitivity analysis.

Conclusions: T2D is associated with impaired pulmonary function, independently of sex, smoking, BMI and geographical region. Longitudinal studies are needed to investigate outcomes for patients with T2D and impaired pulmonary function.

@ERSpublications

T2D is associated with impaired pulmonary function independently of tobacco use. We need to investigate outcomes for T2D patients with impaired pulmonary function. A screening strategy incorporating PFTs must be implemented in T2D patients. https://bit.ly/3iPJy1M

Cite this article as: Díez-Manglano J, Asìn Samper U. Pulmonary function tests in type 2 diabetes: a meta-analysis. ERJ Open Res 2021; 7: 00371-2020 [https://doi.org/10.1183/23120541.00371-2020].

This article has supplementary material available from openres.ersjournals.com.

PROSPERO registry number CRD42020145456.

The study protocol is available in the PROSPERO registry. Immediately following publication, the data will made be available.

Received: 11 June 2020 | Accepted: 30 Sept 2020

Copyright $\odot$ ERS 2021. This article is open access and distributed under the terms of the Creative Commons Attribution Non-Commercial Licence 4.0. 


\section{Introduction}

Diabetes is a chronic disease that affects 463 million people worldwide over the age of 20 years and is expected to affect more than 570 million by 2030 [1]. Diabetes is a leading cause of cardiovascular disease, blindness, kidney failure and lower limb amputation [2]. It is estimated that 4.2 million deaths worldwide were due to type 2 diabetes (T2D) and its complications in 2019 [1].

T2D affects all organs in the human body. It usually develops relatively slowly, and it is frequent the existence of target organ damage when T2D is diagnosed. A number of studies have shown fibrotic changes in the lungs [3] and pulmonary microcirculation disorders in patients with diabetes [4]. There have been persistent attempts investigating abnormal respiratory conditions in general diabetic patients [5-6]. However, pulmonary function impairment has not been well studied in patients with T2D. Although interest in this condition has increased in recent years, the findings of studies reflect high variability. A 2010 meta-analysis by VAN DEN BORST et al. [7] showed an association between T2D and a restrictive pattern. Recently, SAINI et al. [8] have conducted a new systematic review including exclusively English language studies published in PubMed between 2010 and 2018. Both meta-analyses reported data about forced expiratory volume in $1 \mathrm{~s}\left(\mathrm{FEV}_{1}\right)$, forced vital capacity $(\mathrm{FVC})$ and $\mathrm{FEV}_{1} / \mathrm{FVC}$ ratio, and vAN DEN BORST et al. [7] also presented data about diffusing capacity of the lungs for carbon monoxide $\left(D_{\mathrm{LCO}}\right)$.

We hypothesise that the lung may be a target organ of T2D. To contribute to advance the knowledge in this field, we decided to perform a new meta-analysis including literature published in all languages and analysing the influence of publication date, study quality and number of individuals included. Furthermore, as novelty, we determined the influence of sex, tobacco use, geographical area and body mass index (BMI). The aim of this meta-analysis was to investigate the abnormal pulmonary function test results for patients with T2D incorporating the most recent studies. In addition to the parameters reported in the two previous systematic reviews, we included forced expiratory flow between $25 \%$ and $75 \%$ of total lung capacity $\left(\mathrm{FEF}_{25-75 \%}\right)$ and peak expiratory flow $(\mathrm{PEF})$.

\section{Methods}

We designed this meta-analysis to determine the influence of $\mathrm{T} 2 \mathrm{D}$ on the following parameters of pulmonary function tests: $\mathrm{FEV}_{1}, \mathrm{FVC}, \mathrm{FEV}_{1} / \mathrm{FVC}$ ratio, $\mathrm{FEF}_{25-75 \%}, \mathrm{PEF}$ and $D_{\mathrm{LCO}}$.

The protocol for this meta-analysis was recorded in the PROSPERO registry (number CRD42020145456) and was conducted according to the guidelines of the Meta-analysis of Observational Studies in Epidemiology (MOOSE) group.

\section{Data sources and search strategy}

We performed a systematic search in PubMed, EMBASE, The Cochrane Library and Virtual Health Library databases from their inception to August 1, 2019. The search strategy was "(pulmonary function test $\mathrm{OR} \mathrm{FEV}_{1}$ OR FVC OR $D_{\mathrm{LCO}}$ OR PEF OR $\mathrm{FEF}_{25-75}$ ) AND diabetes". We performed an additional search in Google and ResearchGate. The reference lists of the selected studies were screened manually to find more studies.

\section{Study selection}

To be included in this review, the studies had to meet the following inclusion criteria:

1) Presence of a T2D group and a control group without diabetes.

2) Provide values either of $\mathrm{FEV}_{1}, \mathrm{FVC}, \mathrm{PEF}, \mathrm{FEF}_{25-75 \%}, D_{\mathrm{LCO}}$ and/or $\mathrm{FEV}_{1} / \mathrm{FVC}$ ratio for both patient groups.

The exclusion criteria were studies on cystic-fibrosis-related diabetes, type 1 diabetes, studies that did not differentiate between type 1 and T2D, studies that included patients with respiratory diseases as asthma or COPD, studies that did not report data on mean and SD, studies published in predatory journals, conference abstracts, theses and articles published in Chinese language. We considered predatory all journals that appeared in the List of Predatory Journals (https://predatoryjournals.com/journals/). When two studies referred to the same population, in the same period and showed overlapping data, we selected the most recent study for inclusion.

We independently screened the articles by reviewing the titles and abstracts. We recovered the studies that met the inclusion criteria and those with abstracts that lacked crucial information to evaluate the full text. Any discrepancy was resolved by consensus.

When a study's full text was not accessible online or supplemental data were required, we made an attempt to contact the authors by e-mail; unfortunately, these attempts were not successful. 


\section{Quality assessment}

We independently assessed the risk of bias of all the studies included using the Quality Assessment Tool for Observational Cohort and Cross-Sectional Studies (National Heart, Lung and Blood Institute at the National Institutes of Health, USA), available from https://www.nhlbi.nih.gov/health-topics/study-qualityassessment-tools. The tool includes 14 items about objective, population, rate of eligible persons, sample size, exposure, outcomes, blinded assessors, follow-up and confounding variables. The two authors classified the studies as good, fair or poor. Any discrepancy was resolved by consensus. We considered a study as poor when T2D patients and controls were not selected from the same population or in a different time or place, and fair when we cannot determine this and there were doubts about a selection bias. All studies were included in the meta-analysis; however, we conducted a sensitivity study only on those studies of good quality.

\section{Data extraction}

From each included study, we extracted the following information: first author, year of publication, country, sample size, patient age, sex, BMI, tobacco use, T2D duration, fasting blood glucose, glycated haemoglobin and microangiopathy. The extracted results were $\mathrm{FEV}_{1}(\mathrm{~L})$, percentage of predicted (\%)

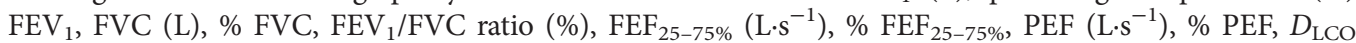
$\left(\mathrm{mL} \cdot \mathrm{min}^{-1} \cdot \mathrm{mmHg}^{-1}\right)$ and $\% D_{\mathrm{LCO}}$. Whenever the T2D or control group was divided into subgroups, a pooled mean and SD for these combined subgroups was calculated.

\section{Data synthesis and statistical analysis}

We performed the statistical analysis using Review Manager version 5.3 (Cochrane Collaboration, Baltimore, MD, USA). The results are expressed as mean difference (MD) with $95 \%$ confidence interval (CI). Throughout the analysis, we applied the inverse variance method with a random-effects model. To assess the heterogeneity and inconsistency between the studies, we employed the tau squared and $\mathrm{I}^{2}$ statistics. Data with $\mathrm{p} \geqslant 0.10$ and $\mathrm{I}^{2} \leqslant 50 \%$ were defined as low heterogeneity. We evaluated the publication bias with a funnel plot. We planned a meta-regression analysis by subgroup according to sex, geographical area, tobacco use and BMI. We performed a sensitivity analysis by applying a fixed-effects model and calculating the effect estimates according to publication date, size of T2D group and study quality and by eliminating the study with the greatest weight on the effect. We established three categories of publication year, before 2000, 2000-2009 and 2010-2019, and two categories of T2D group size, $<50$ and $\geqslant 50$ patients. For the sensitivity analysis according study quality, we calculated the effect estimates in two ways: including only the good quality studies; and including all studies adding predatory journals and grey literature.

\section{Results}

\section{Study selection}

We identified 17662 records. Figure 1 shows the study selection flowchart. Our initial search strategy produced 17549 articles. With the manual search of the reference lists and the additional search in Google and ResearchGate, we added 115 articles. After eliminating the duplicated and irrelevant articles, we were left with 263 articles. We excluded 191 articles for the following reasons: 62 had no control group, 49 included patients with types 1 and 2 diabetes without differentiating them, 30 provided insufficient numerical data to be included in the meta-analysis, 26 originated from predatory journals, 10 presented overlapping data, six came from grey literature (theses and proceedings), four were in Chinese language, one included patients with respiratory diseases, two were meta-analyses and one was an editorial. There was no interrater agreement in study selection and consensus was necessary for eight studies. Furthermore, the full text of six papers was not found (supplementary material). Ultimately, we included 66 studies in the meta-analysis [9-74], one longitudinal, two case-control and 63 cross-sectional ones. From the longitudinal study, we extracted only the baseline pulmonary function test data.

\section{Study characteristics}

Table 1 lists the characteristics of the included studies, which were published between 1991 and 2018 . Three studies were conducted in Africa, 11 in America, 33 in Asia, 18 in Europe and one in Oceania. Fifty-eight studies were written in English, 4 in Turkish, 2 in Spanish, one in German and one in Japanese. After the quality assessment, we classified 54 studies as good, six as fair and six as poor. The interrater agreement was full. A total of 59511 participants were included, 11134 in the T2D group and 48377 in the control group. The age range of T2D patients was $39.8-79$ years, and $35.1 \%$ were women.

\section{Pulmonary function tests}

We provide here data on predicted percentages of pulmonary function tests. Data about absolute values are reported in supplementary material. 


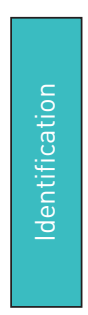

Records identified through database searching

( $n=17549$ )

PubMed: 4228

Embase: 9528

Virtual Health Library: 3497

Cochrane Library Trials: 296
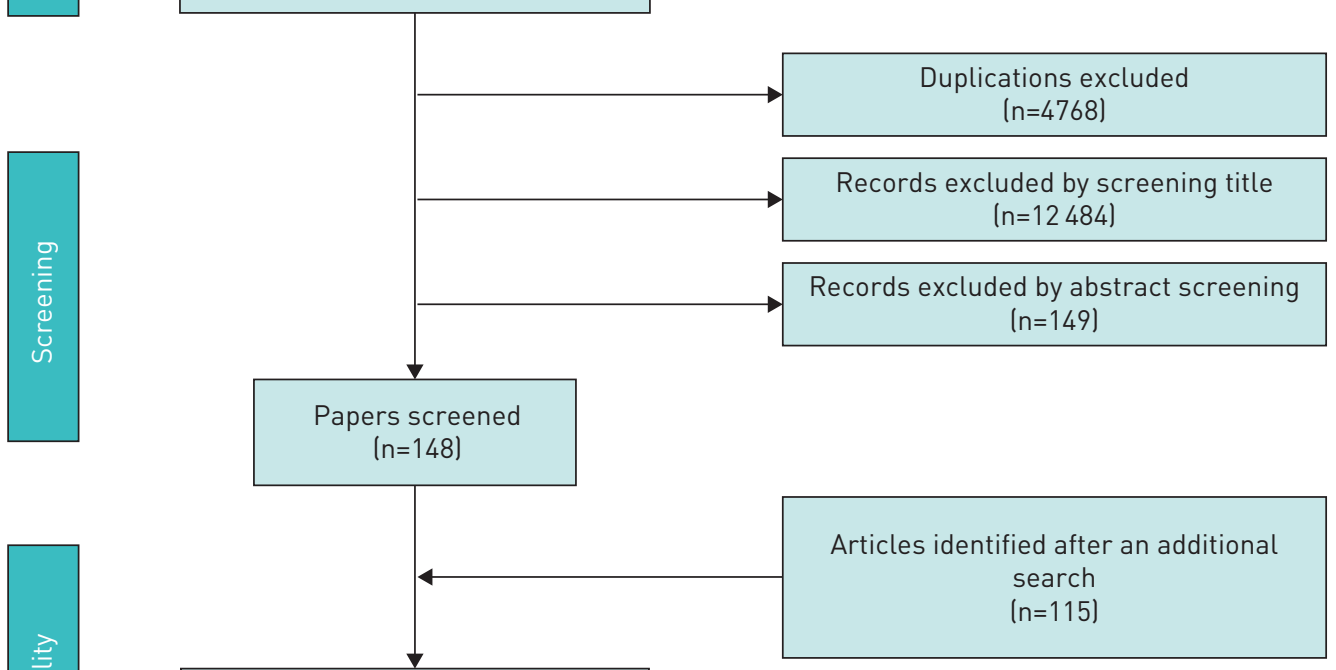

Fult-text screening for eligibility ( $n=263)$

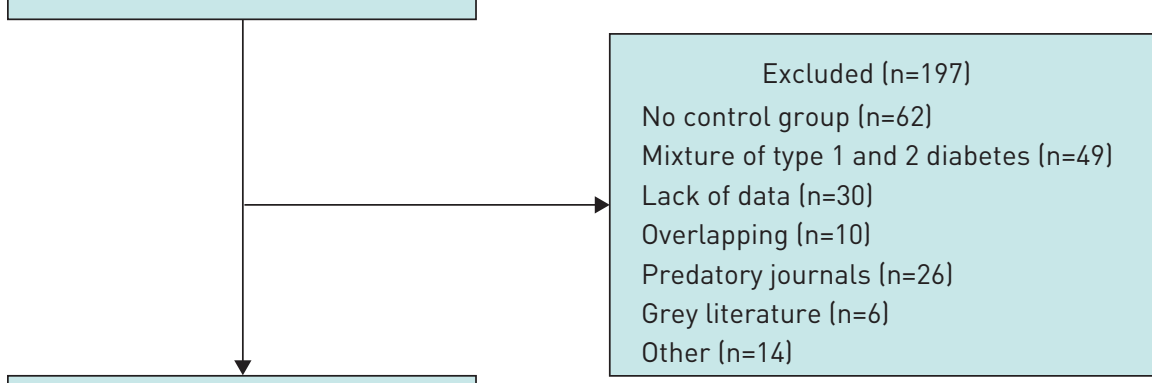

Papers included in meta-analysis $(n=66)$

FIGURE 1 Flowchart of included studies.

FEV 1

A total of 41 studies included data on $\% \mathrm{FEV}_{1}$, and 34 included data on $\mathrm{FEV}_{1}$ (L). Figure $2 \mathrm{a}$ and figure $\mathrm{S} 1$ (supplementary material) show the comparison forest plot. The pooled effect estimates for the patients with T2D were -7.15 ( $95 \% \mathrm{CI}-8.27$ to -6.03 ; $\mathrm{p}<0.0001)$ for $\% \mathrm{FEV}_{1}$ and -0.34 (95\% CI -0.42 to -0.27 ; $\mathrm{p}<0.0001)$ for $\mathrm{FEV}_{1}(\mathrm{~L})$.

FVC

A total of 35 studies included data on \% FVC, and 23 included data on FVC (L). Figure 2b and figure S2 (supplementary material) show the effect estimates. The pooled estimates for the patients with T2D were -9.21 (95\% CI -11.15 to -7.26 ; $\mathrm{p}<0.0001)$ for $\% \mathrm{FVC}$ and $-0.36(95 \% \mathrm{CI}-0.43$ to $-0.29 ; \mathrm{p}<0.0001)$ for FVC (L).

\section{$F E V_{1} / F V C$ ratio}

A total of 45 studies included data on the $\mathrm{FEV}_{1} / \mathrm{FVC}$ ratio (\%). Figure 3 shows the comparison forest plot. The pooled effect estimate for the patients with T2D was -0.27 (95\% CI -1.63 to $1.08 ; \mathrm{p}<0.69$ ). 
TABLE 1 Characteristics of the included studies and patients with type 2 diabetes

\begin{tabular}{|c|c|c|c|c|c|c|c|c|c|c|c|c|}
\hline Ref. & $\begin{array}{l}\text { Study author, } \\
\text { year }\end{array}$ & $\begin{array}{c}\text { Country } \\
\text { (continent) }\end{array}$ & $\begin{array}{l}\text { DM group } \\
\text { size (men/ } \\
\text { women) }\end{array}$ & $\begin{array}{l}\text { Mean } \\
\text { age } \\
\text { years }\end{array}$ & $\begin{array}{c}\text { Smokers } \\
\%\end{array}$ & $\begin{array}{c}\mathrm{BMI} \\
\mathrm{kg} \cdot \mathrm{m}^{-2}\end{array}$ & $\begin{array}{l}\text { Fasting blood } \\
\text { glucose } \\
\text { mmol. } \mathrm{L}^{-1}\end{array}$ & $\begin{array}{c}\text { Glycated } \\
\text { Hb \% }\end{array}$ & $\begin{array}{c}\text { T2D } \\
\text { duration } \\
\text { years }\end{array}$ & $\begin{array}{c}\text { Patients with } \\
\text { microangiopathy } \\
\%\end{array}$ & $\begin{array}{c}\text { Pulmonary function } \\
\text { tests }\end{array}$ & $\begin{array}{l}\text { Study } \\
\text { quality }\end{array}$ \\
\hline [9] & MatsubARA, 1991 & Japan (As) & $53(29 / 23)$ & 58.0 & NR & NR & NR & 9.5 & 7.4 & NR & $\mathrm{FEV}_{1}, D_{\text {LCO }}$ & Fair \\
\hline [10] & $\begin{array}{l}\text { LARA-RODRÍGUEz, } \\
1995\end{array}$ & $\begin{array}{l}\text { Venezuela } \\
\text { (Am) }\end{array}$ & $12(7 / 5)$ & 47.0 & 0 & NR & NR & NR & NR & 83 & $\begin{array}{l}\mathrm{FEV}_{1}, \mathrm{FVC} \\
\mathrm{FEV}_{1} / \mathrm{FVC}\end{array}$ & Fair \\
\hline [11] & $\begin{array}{l}\text { BARRETT-CONNOR, } \\
1996\end{array}$ & USA (Am) & $139(71 / 68)$ & 75.9 & 5.7 & 26.3 & 6.94 & NR & NR & NR & $\begin{array}{c}\mathrm{FEF}_{25-75 \%}, D_{\mathrm{LCO}} \\
\mathrm{FEV}_{1}, \mathrm{FVC}\end{array}$ & Good \\
\hline [12] & КАТОН, 1996 & Japan (As) & 19 (10/9) & 50.5 & NR & 27.8 & 7.49 & 7.5 & NR & NR & $\mathrm{FEV}_{1}$ & Fair \\
\hline [13] & ISOTANI, 1999 & Japan (As) & $54(23 / 31)$ & 54.9 & 0 & 22.2 & 9.77 & 9 & 11.0 & 68.5 & $\mathrm{FEV}_{1}, D_{\mathrm{LCO}}$ & Good \\
\hline [14] & BENBASSAT, 2001 & Israel (As) & $12(8 / 4)$ & 60 & 0 & 29.6 & NR & 9.0 & 12.8 & 33 & $\begin{array}{c}\mathrm{FEV}_{1}, \mathrm{FVC} \\
\mathrm{FEF}_{25-75 \%}, D_{\mathrm{LCo}}\end{array}$ & Good \\
\hline [15] & ZAMARRÓN, 2001 & Spain (Eu) & $31(5 / 26)$ & 71.1 & 0 & NR & NR & NR & NR & NR & $\begin{array}{c}\mathrm{FEV}_{1}, \mathrm{FVC}, \\
\mathrm{FEV}_{1} / \mathrm{FVC}, \\
\mathrm{FEF}_{25-75 \%} D_{\mathrm{LCO}}\end{array}$ & Good \\
\hline [16] & ARI, 2002 & Turkey (Eu) & $25(5 / 20)$ & 55.6 & 0 & 25.8 & NR & 7.5 & 9.4 & 25 & $\begin{array}{l}\mathrm{FEV}_{1}, \mathrm{FVC} \\
\mathrm{FEV}_{1} / \mathrm{FVC}\end{array}$ & Good \\
\hline [17] & Guazzı, 2002 & Italy (Eu) & $15(8 / 7)$ & 62.3 & 0 & NR & 7.6 & 6.1 & NR & NR & $\begin{array}{c}\mathrm{FEF}_{25-75 \%}, \mathrm{PEF}, D_{\mathrm{LCO}} \\
\mathrm{FEV}_{1}, D_{\mathrm{LCO}}\end{array}$ & Good \\
\hline [18] & MAIOLO, 2002 & Italy (Eu) & $12(0 / 12)$ & 50.3 & 0 & 32.6 & 11.43 & NR & NR & NR & $\begin{array}{c}\mathrm{FEV}_{1}, \mathrm{FVC} \\
\mathrm{FEV}_{1} / \mathrm{FVC}, \mathrm{PEF}\end{array}$ & Good \\
\hline [19] & Boulbou, 2003 & Greece (Eu) & 33 & NR & 0 & 27.3 & NR & NR & NR & NR & $\begin{array}{c}\mathrm{FEV}_{1}, \mathrm{FVC} \\
\mathrm{FEV}_{1} / \mathrm{FVC}, D_{\mathrm{LCO}}\end{array}$ & Good \\
\hline [20] & GUVENER, 2003 & Turkey (Eu) & $25(9 / 16)$ & 56.3 & 0 & 29.9 & NR & 7.4 & 5.8 & 63.6 & $\begin{array}{c}\mathrm{FEV}_{1}, \mathrm{FVC}, \mathrm{PEF}, \\
D_{\mathrm{LCO}}\end{array}$ & Good \\
\hline [21] & Melo, 2003 & Brazil (Am) & $17(8 / 9)$ & 47.0 & 0 & NR & NR & NR & 7.5 & 47 & $\begin{array}{c}\mathrm{FEV}_{1}, \mathrm{FVC} \\
\mathrm{FEV}_{1} / \mathrm{FVC}, \mathrm{FEF}_{25-75}\end{array}$ & Good \\
\hline [22] & LAU, 2004 & China (As) & $40(26 / 14)$ & 49.8 & 10 & 25.9 & NR & 7.9 & 7.8 & 50 & $\begin{array}{c}\mathrm{FEV}_{1}, \mathrm{FVC} \\
\mathrm{FEV}_{1} / \mathrm{FVC}, D_{\mathrm{LCO}}\end{array}$ & Poor \\
\hline [23] & SINHA, 2004 & India (As) & $29(21 / 8)$ & 46.7 & 0 & 24.9 & 14.54 & 8.6 & 4.4 & 41.4 & $\begin{array}{c}\mathrm{FEV}_{1}, \mathrm{FVC}, \mathrm{PEF}, \\
D_{\mathrm{LCO}}\end{array}$ & Good \\
\hline [24] & WEISBROD, 2005 & Australia (Oc) & $8(5 / 3)$ & 56.2 & 0 & 29.9 & 9.1 & 7.9 & 5.1 & 0 & $\mathrm{FEV}_{1}, \mathrm{FVC}, \mathrm{FEV}_{1} / \mathrm{FVC}$ & Good \\
\hline [25] & Meo, 2006 & $\begin{array}{l}\text { Saudi Arabia } \\
\text { (As) }\end{array}$ & 32 (32/0) & 52.6 & 0 & NR & NR & NR & 10 & NR & $\begin{array}{l}\mathrm{FEV}_{1}, \mathrm{FVC} \\
\mathrm{FEV}_{1} / \mathrm{FVC}\end{array}$ & Good \\
\hline [26] & $\begin{array}{l}\text { ORTIZ-AgUiRRE, } \\
2006\end{array}$ & Mexico (Am) & $144(54 / 90)$ & 57.7 & 36.8 & 28.8 & 9.16 & NR & 9.2 & NR & $\begin{array}{c}\mathrm{FEF}_{25-75 \%,} \text { PEF } \\
\mathrm{FEV}_{1}, \mathrm{FVC} \\
\mathrm{FEV}_{1} / \mathrm{FV}, \mathrm{PEF}\end{array}$ & Poor \\
\hline [27] & OZSAHIN, 2006 & Turkey (Eu) & $25(6 / 19)$ & 55 & 0 & 24.3 & NR & NR & 9.3 & 92 & $D_{\mathrm{LCO}}$ & Fair \\
\hline [28] & Chance, 2008 & USA (Am) & $69(38 / 31)$ & 46.1 & 0 & 31.1 & NR & 8.3 & 7.8 & 38 & $\mathrm{FEV}_{1}, \mathrm{FVC}, \mathrm{FEV}_{1} / \mathrm{FVC}$ & Good \\
\hline [29] & DenNis, 2008 & Colombia (Am) & $\begin{array}{c}262(107 / \\
155)\end{array}$ & 50.9 & 15.3 & NR & NR & NR & NR & NR & $\mathrm{FEV}_{1}, \mathrm{FVC}, \mathrm{FEV}_{1} / \mathrm{FVC}$ & Good \\
\hline
\end{tabular}


TABLE 1 Continued

\begin{tabular}{|c|c|c|c|c|c|c|c|c|c|c|c|c|}
\hline Ref. & $\begin{array}{l}\text { Study author, } \\
\text { year }\end{array}$ & $\begin{array}{c}\text { Country } \\
\text { (continent) }\end{array}$ & $\begin{array}{l}\text { DM group } \\
\text { size (men/ } \\
\text { women) }\end{array}$ & $\begin{array}{c}\text { Mean } \\
\text { age } \\
\text { years }\end{array}$ & $\begin{array}{c}\text { Smokers } \\
\%\end{array}$ & $\begin{array}{c}\mathrm{BMI} \\
\mathrm{kg} \cdot \mathrm{m}^{-2}\end{array}$ & $\begin{array}{l}\text { Fasting blood } \\
\text { glucose } \\
\text { mmol. } \mathrm{L}^{-1}\end{array}$ & $\begin{array}{c}\text { Glycated } \\
\mathrm{Hb} \%\end{array}$ & $\begin{array}{c}\text { T2D } \\
\text { duration } \\
\text { years }\end{array}$ & $\begin{array}{c}\text { Patients with } \\
\text { microangiopathy } \\
\%\end{array}$ & $\begin{array}{l}\text { Pulmonary function } \\
\text { tests }\end{array}$ & $\begin{array}{l}\text { Study } \\
\text { quality }\end{array}$ \\
\hline [30] & $K_{A B I T Z,} 2008$ & Germany (Eu) & $21(21 / 0)$ & 63.6 & NR & 28.5 & NR & 7.3 & 12.9 & 52.4 & $\mathrm{FEV}_{1}, \mathrm{FVC}, \mathrm{FEV}_{1} / \mathrm{FVC}$ & Good \\
\hline [31] & ҮЕН, 2008 & USA (Am) & $\begin{array}{c}1100(528 / \\
572)\end{array}$ & 55 & 19 & 30.9 & NR & NR & NR & NR & $\mathrm{FEV}_{1}, \mathrm{FVC}, \mathrm{FEV}_{1} / \mathrm{FVC}$ & Good \\
\hline [32] & AL, 2009 & $\begin{array}{l}\text { Bangladesh } \\
\text { (As) }\end{array}$ & $60(60 / 0)$ & 51.8 & 0 & 21.3 & NR & 6.8 & 10.6 & NR & $\mathrm{FEV}_{1}, \mathrm{FVC}, \mathrm{FEV}_{1} / \mathrm{FVC}$ & Good \\
\hline [33] & SALER, 2009 & Turkey (Eu) & 68 (19/49) & 52.4 & 0 & 27.0 & NR & 7.4 & 7.6 & 44 & $D_{\mathrm{LCO}}$ & Good \\
\hline [34] & VERMA, 2009 & India (As) & $50(30 / 20)$ & 50.2 & 0 & NR & NR & NR & NR & NR & $\begin{array}{l}\mathrm{FEV}_{1}, \mathrm{FVC}, \\
\mathrm{FEV} / \mathrm{FVC}\end{array}$ & Good \\
\hline [35] & AgARWAL, 2010 & India (As) & 30 (17/13) & 44.6 & 0 & 22.0 & NR & 8.7 & 5.4 & 50 & $\begin{array}{c}\mathrm{FEF}_{25-75 \%,} \text { PEF } \\
\mathrm{FEV}_{1}, \mathrm{FVC}, \\
\mathrm{FEF}_{25-75 \%} \text {, PEF, } D_{\mathrm{LCO}}\end{array}$ & Good \\
\hline [36] & ALI, 2010, & $\begin{array}{l}\text { Bangladesh } \\
\text { (As) }\end{array}$ & $60(60 / 0)$ & 51.8 & 0 & 21.3 & NR & 6.8 & 10.6 & NR & $\mathrm{FEF}_{25-75 \%}, \mathrm{PEF}$ & Good \\
\hline [37] & LecuBe, 2010 & Spain (Eu) & $25(0 / 25)$ & 44.0 & 0 & 49.2 & 8.6 & 7.5 & NR & 16 & $\begin{array}{c}\text { FEV }, \text { FVC, } \\
\mathrm{FEV}_{1} / \mathrm{FVC}^{-}, \mathrm{FEF}_{25-75 \%}\end{array}$ & Good \\
\hline [38] & OzoH, 2010 & Nigeria (Af) & $101(47 / 54)$ & 46.1 & 0 & 28.3 & NR & 7.8 & $1 \mathrm{~m}-18 \mathrm{y}$ & NR & $\begin{array}{l}\text { FEV }, \text { FCC, } \\
\text { FEV }_{1} / \text { FVC, PEF }\end{array}$ & Good \\
\hline [39] & $\begin{array}{l}\text { BuYUKHATIPOGLU, } \\
2011\end{array}$ & Turkey (Eu) & $80(40 / 40)$ & 47.8 & 0 & 26.7 & 10.77 & 9.3 & 5 & 50 & $D_{\mathrm{LCO}}$ & Good \\
\hline [40] & CEylan, 2011 & Turkey (Eu) & $37(16 / 21)$ & 39.8 & 0 & NR & NR & 8.2 & 7 & NR & $\begin{array}{l}\mathrm{FEV}_{1}, \mathrm{FVC} \\
\mathrm{FEV}_{1} / \mathrm{FVC}\end{array}$ & Good \\
\hline [41] & DHARWADKAR, 2011 & India (As) & $40(25 / 15)$ & 52.3 & 0 & 22.7 & 8.2 & NR & 6.4 & NR & $\begin{array}{l}\mathrm{FEF}_{25-75 \%} \text {, PEF, } D_{\text {LCO }} \\
\text { FEV } 1 \text {, FVC, PEEF }\end{array}$ & Poor \\
\hline [42] & KIM, 2011 & $\begin{array}{l}\text { South Korea } \\
\text { (As) }\end{array}$ & $\begin{array}{c}2745(2168 / \\
577)\end{array}$ & 55 & 29 & 25.1 & NR & NR & NR & NR & $\mathrm{FEV}_{1}, \mathrm{FVC}, \mathrm{FEV}_{1} / \mathrm{FVC}$ & Good \\
\hline [43] & KLEIN, 2011 & USA (Am) & 76 (33/43) & 63.1 & 29 & 34.2 & NR & NR & 6.7 & NR & $\mathrm{FEV}_{1}, \mathrm{FVC}$ & Good \\
\hline [44] & AL-HABBO, 2012 & Iraq (As) & $45(26 / 19)$ & 46.7 & NR & NR & NR & NR & NR & NR & $\begin{array}{l}\mathrm{FEV}_{1}, \mathrm{FVC} \\
\mathrm{FEV}_{1} / \mathrm{FVC}\end{array}$ & Good \\
\hline [45] & KLEIN, 2012 & USA (Am) & $\begin{array}{l}303(178 / \\
125)\end{array}$ & 61.7 & 29.4 & 31.4 & NR & NR & NR & NR & $\begin{array}{c}\mathrm{FEF}_{25-75 \%,} \mathrm{PEF} \\
D_{\mathrm{LCO}}\end{array}$ & Good \\
\hline [46] & KLEIN, 2012 & USA (Am) & $\begin{array}{c}560(314 / \\
260)\end{array}$ & 62.0 & 27 & 31.7 & NR & NR & NR & NR & $\mathrm{FEV}_{1}, \mathrm{FVC}, D_{\mathrm{LCO}}$ & Good \\
\hline [47] & NANDHINI, 2012 & India (As) & $45(30 / 15)$ & 47.1 & NR & NR & 7.1 & NR & 6.3 & NR & $\begin{array}{l}\mathrm{FEV}_{1}, \mathrm{FVC}, \\
\mathrm{FEV}_{1} / \mathrm{FVC}\end{array}$ & Good \\
\hline [48] & $\begin{array}{l}\text { ABd-EL-AzEem, } \\
2013\end{array}$ & Egypt (Af) & 30 & NR & 0 & NR & NR & NR & NR & NR & $\begin{array}{c}\mathrm{FEF}_{25-75 \%,} \text { PEF } \\
\mathrm{FEV}_{1}, \mathrm{FVC}, \\
\mathrm{FEV}_{1} / \mathrm{FVC}, \\
\mathrm{FEF}_{25-75 \%}, \mathrm{PEF}, D_{\mathrm{LCO}}\end{array}$ & Good \\
\hline
\end{tabular}




\begin{tabular}{|c|c|c|c|c|c|c|c|c|c|c|c|c|}
\hline Ref. & $\begin{array}{l}\text { Study author, } \\
\text { year }\end{array}$ & $\begin{array}{c}\text { Country } \\
\text { (continent) }\end{array}$ & $\begin{array}{l}\text { DM group } \\
\text { size (men/ } \\
\text { women) }\end{array}$ & $\begin{array}{l}\text { Mean } \\
\text { age } \\
\text { years }\end{array}$ & $\begin{array}{c}\text { Smokers } \\
\%\end{array}$ & $\begin{array}{c}\mathrm{BMI} \\
\mathrm{kg} \cdot \mathrm{m}^{-2}\end{array}$ & $\begin{array}{l}\text { Fasting blood } \\
\text { glucose } \\
\mathrm{mmol} \cdot \mathrm{L}^{-1}\end{array}$ & $\begin{array}{l}\text { Glycated } \\
\text { Hb \% }\end{array}$ & $\begin{array}{l}\text { T2D } \\
\text { duration } \\
\text { years }\end{array}$ & $\begin{array}{c}\text { Patients with } \\
\text { microangiopathy } \\
\%\end{array}$ & $\begin{array}{l}\text { Pulmonary function } \\
\text { tests }\end{array}$ & $\begin{array}{l}\text { Study } \\
\text { quality }\end{array}$ \\
\hline [49] & AKBER, 2013 & Iraq (As) & $63(28 / 35)$ & 51.0 & 0 & 30.0 & NR & NR & NR & NR & $\mathrm{FEV}_{1}, \mathrm{FVC}, \mathrm{FEV}_{1} / \mathrm{FVC}$ & Good \\
\hline [50] & ALKINANY, 2013 & Iraq (As) & $60(60 / 0)$ & $40-60$ & 0 & NR & NR & NR & NR & NR & $\mathrm{FEV}_{1}, \mathrm{FVC}$ & Poor \\
\hline [51] & $\begin{array}{l}\text { ANANDHALAKSHMI, } \\
2013\end{array}$ & India (As) & 30 & 44.8 & 0 & 26.1 & NR & 6.8 & 7.0 & NR & $\begin{array}{l}F E V_{1}, F V C \\
F E V_{1} / F V C\end{array}$ & Good \\
\hline [52] & APARNA, 2013 & India (As) & $40(22 / 18)$ & 49 & 0 & 25.2 & NR & NR & NR & NR & $\begin{array}{c}\text { FEF }_{25-75 \%}, \mathrm{PEF}, U_{\text {LCO }} \\
\text { FEV }_{1}, \mathrm{FVC} \text {, } \\
\mathrm{FEV}_{1} / \mathrm{FVC}, \mathrm{PEF}\end{array}$ & Fair \\
\hline [53] & RAJANI, 2013 & India (As) & $40(19 / 21)$ & 46 & 0 & NR & 10.88 & 7.0 & NR & NR & $\begin{array}{l}\mathrm{FEV}_{1}, \mathrm{FVC} \\
\mathrm{FEV}_{1} / \mathrm{FVC}\end{array}$ & Good \\
\hline [54] & ShafieE, 2013 & Iran (As) & $80(31 / 49)$ & 53.6 & 0 & 28.8 & NR & 8.4 & 9.8 & 50 & $\begin{array}{l}\mathrm{FEF}_{25-75 \%}, \mathrm{PEF} \\
\mathrm{FEV}_{1}, \mathrm{FVC} \\
\mathrm{FEV}_{1} / \mathrm{FVC}, \mathrm{PEF}\end{array}$ & Good \\
\hline [55] & SHAH, 2013 & India (As) & $60(60 / 0)$ & 53.9 & 0 & NR & NR & 7.1 & 6.6 & NR & $\begin{array}{c}\mathrm{FEV}_{1}, \mathrm{FVC} \\
\mathrm{FEV}_{1} / \mathrm{FVC} \\
\mathrm{FEF}_{25-75 \%}, \mathrm{PEF}\end{array}$ & Good \\
\hline [56] & HuANG, 2014 & China (As) & $\begin{array}{c}292(181 / \\
111)\end{array}$ & 66.8 & 0 & 23.9 & 8.55 & NR & 5.2 & NR & $\mathrm{FEV}_{1}, \mathrm{FVC}, \mathrm{FEV} \mathrm{V}_{1} / \mathrm{FVC}$ & Good \\
\hline [57] & JAMATIA, 2014 & India (As) & $30(19 / 11)$ & 57.7 & 0 & 23.5 & 10.71 & 7.93 & $>2$ & 0 & $\begin{array}{l}\mathrm{FEV}_{1}, \mathrm{FVC} \\
\mathrm{FEV}_{1} / \mathrm{FVC}\end{array}$ & Good \\
\hline [58] & Uz-Zaman, 2014 & India (As) & 60 & 44.6 & 0 & 24.4 & 9.93 & 7.1 & NR & NR & $\begin{array}{c}\mathrm{FEF}_{25-75 \%}, \mathrm{PEF} \\
\mathrm{FEV}_{1}, \mathrm{FVC} \\
\mathrm{FEV}_{1} / \mathrm{FVC}\end{array}$ & Good \\
\hline [59] & ZINELDIN, 2015 & Egypt (Af) & $45(45 / 0)$ & 51.1 & 0 & 24.5 & NR & 7.5 & 7.6 & NR & $\begin{array}{c}\mathrm{FEF}_{25-75 \%}, \mathrm{PEF}, \mathrm{D}_{\mathrm{LCO}} \\
\mathrm{FEV}_{1}, \mathrm{FVC} \\
\mathrm{FEV}_{1} / \mathrm{FVC}, \mathrm{PEF}\end{array}$ & Good \\
\hline [60] & BuchmANn, 2016 & Germany (Eu) & $91(49 / 42)$ & 67.9 & 9.6 & 29.2 & NR & NR & NR & NR & $\mathrm{FEV}_{1}, \mathrm{FVC}, \mathrm{FEV} \mathrm{V}_{1} / \mathrm{FVC}$ & Good \\
\hline [61] & KAUR, 2016 & India (As) & 50 & NR & 0 & NR & NR & NR & NR & NR & $\begin{array}{c}\mathrm{FEV}_{1}, \mathrm{FVC} \\
\mathrm{FEV}_{1} / \mathrm{FVC}, \mathrm{PEF}\end{array}$ & Good \\
\hline [62] & KuMAR, 2016 & India (As) & 40 & 50.7 & 0 & 25.4 & 10.6 & 8.5 & 11.1 & 50 & $\begin{array}{c}\mathrm{FEV}_{1}, \mathrm{FVC} \\
\mathrm{FEV}_{1} / \mathrm{FVC}, D_{\mathrm{LCO}}\end{array}$ & Poor \\
\hline [63] & CARON, 2017 & Canada (Am) & $10(10 / 0)$ & 55 & NR & 30.0 & 6.5 & 6.1 & 1.25 & 0 & $\mathrm{FEV}_{1}, \mathrm{FVC}, D_{\mathrm{LCO}}$ & Good \\
\hline [64] & KHAFAIE, 2017 & Iran (As) & $\begin{array}{c}347(268 / \\
79)\end{array}$ & 54.6 & 21 & 26.7 & 7.98 & 8.8 & NR & NR & $\mathrm{FEV}_{1}, \mathrm{FVC}, \mathrm{FEV}_{1} / \mathrm{FVC}$ & Poor \\
\hline [65] & k, 2017 & $\begin{array}{c}\text { South Korea } \\
\text { (As) }\end{array}$ & $\begin{array}{c}1431(814 / \\
617)\end{array}$ & 59.1 & 20 & 25.4 & 7.54 & 7.3 & 4.7 & NR & $\mathrm{FEV}_{1}, \mathrm{FVC}, \mathrm{FEV}_{1} / \mathrm{FVC}$ & Good \\
\hline [66] & LóPEZ-CANo, 2017 & Spain (Eu) & $49(12 / 37)$ & 51.3 & 0 & 42.0 & 9.2 & 8.0 & NR & NR & $\begin{array}{l}\mathrm{FEV}_{1}, \mathrm{FVC} \\
\mathrm{FEF}_{25-75 \%}\end{array}$ & Good \\
\hline [67] & NidHIANAND, 2017 & India (As) & $100(57 / 43)$ & 46.6 & 0 & NR & NR & NR & NR & NR & $\begin{array}{c}\mathrm{FEV}_{1}, \mathrm{FVC} \\
\mathrm{FEV}_{1} / \mathrm{FVC}, \mathrm{PEF}\end{array}$ & Good \\
\hline [68] & SHERGILL, 2017 & India (As) & $50(50 / 0)$ & 52.6 & NR & 23.7 & NR & NR & NR & NR & $\begin{array}{c}\text { FEV }_{1}, F V C \\
\text { FEV }_{1} / \text { FVC, PEF }\end{array}$ & Good \\
\hline
\end{tabular}




\begin{tabular}{|c|c|c|c|c|c|c|c|c|c|c|c|c|}
\hline Ref. & $\begin{array}{l}\text { Study author, } \\
\text { year }\end{array}$ & $\begin{array}{c}\text { Country } \\
\text { (continent) }\end{array}$ & $\begin{array}{l}\text { DM group } \\
\text { size (men/ } \\
\text { women) }\end{array}$ & $\begin{array}{l}\text { Mean } \\
\text { age } \\
\text { years }\end{array}$ & $\begin{array}{c}\text { Smokers } \\
\%\end{array}$ & $\begin{array}{c}\mathrm{BMI} \\
\mathrm{kg} \cdot \mathrm{m}^{-2}\end{array}$ & $\begin{array}{l}\text { Fasting blood } \\
\text { glucose } \\
\mathrm{mmol} \cdot \mathrm{L}^{-1}\end{array}$ & $\begin{array}{c}\text { Glycated } \\
\text { Hb \% }\end{array}$ & $\begin{array}{c}\text { T2D } \\
\text { duration } \\
\text { years }\end{array}$ & $\begin{array}{c}\text { Patients with } \\
\text { microangiopathy } \\
\%\end{array}$ & $\begin{array}{l}\text { Pulmonary function } \\
\text { tests }\end{array}$ & $\begin{array}{l}\text { Study } \\
\text { quality }\end{array}$ \\
\hline [69] & $\mathrm{T}_{\mathrm{Al}}, 2017$ & China (As) & $63(34 / 29)$ & 53.0 & 0 & 27.7 & 7.9 & 8.0 & 7.8 & NR & $\begin{array}{c}\mathrm{FEV}_{1}, \mathrm{FVC} \\
\mathrm{FEV}_{1} / \mathrm{FVC}, D_{\mathrm{LCO}}\end{array}$ & Good \\
\hline [70] & WiLMS, 2017 & $\begin{array}{l}\text { Switzerland } \\
\text { (Eu) }\end{array}$ & $65(19 / 46)$ & 46.9 & 27.7 & 44.0 & 8.9 & 7.9 & NR & NR & $\mathrm{FEV}_{1}, \mathrm{FEV}_{1} / \mathrm{FVC}$ & Fair \\
\hline [71] & OKUR, 2018 & Turkey (Eu) & $58(15 / 43)$ & 53.3 & NR & 31.7 & 10.27 & 8.2 & 9.9 & NR & $\begin{array}{c}\mathrm{FEV}_{1}, \mathrm{FVC}, \\
\mathrm{FEV}_{1} / \mathrm{FVC} \\
\mathrm{FEF}_{25-75 \%}, \mathrm{PEF}\end{array}$ & Good \\
\hline [72] & RohLING, 2018 & Germany (Eu) & $34(21 / 13)$ & 53.0 & 26.5 & 30.8 & NR & 6.4 & 0.35 & NR & $\mathrm{FEV}_{1}, \mathrm{FVC}$ & Good \\
\hline [73] & TAYARAMI, 2018 & Iran (As) & 50 & 58.3 & 0 & NR & NR & NR & NR & NR & $\begin{array}{l}\mathrm{FEV}_{1}, \mathrm{FVC} \\
\mathrm{FEV}_{1} / \mathrm{FVC}\end{array}$ & Good \\
\hline [74] & $\begin{array}{l}\text { VAn EetVelde, } \\
2018\end{array}$ & Belgium (Eu) & $110(39 / 71)$ & 79 & NR & 295 & NR & 6.7 & 10.3 & 74.5 & $\begin{array}{c}\mathrm{FEF}_{25-75 \%,} \text { PEF } \\
\text { PEF }\end{array}$ & Good \\
\hline
\end{tabular}

DM: diabetes mellitus; BMI: body mass index; Hb: haemoglobin; T2D: type-2 diabetes; FEV ${ }_{1}$ forced expiratory volume in $1 \mathrm{~s}$; As: Asia; FVC: forced vital capacity; $D_{\mathrm{LCo}}$ : diffusion capacity of the lungs for carbon monoxide; NR: not reported; Am: America; Af: Africa; FEF $25-75 \%$ : forced expiratory flow between $25-75 \%$ of FVC; Eu: Europe; m: months; PEF: peak expiratory flow; Oc: Oceania; y: years. 
a)

\begin{tabular}{|c|c|c|c|c|c|c|c|c|c|}
\hline \multirow[t]{2}{*}{ Study or subgroup } & \multicolumn{2}{|c|}{ Type-2 diabetes } & \multicolumn{2}{|c|}{ Control } & \multirow[t]{2}{*}{ Weight } & \multirow{2}{*}{$\begin{array}{c}\text { Mean difference } \\
\text { IV, random }(95 \% \mathrm{CI})\end{array}$} & \multirow{2}{*}{\multicolumn{3}{|c|}{$\begin{array}{c}\text { Mean difference } \\
\text { IV, random }(95 \% \mathrm{CI})\end{array}$}} \\
\hline & Mean \pm SD & Total & Mean \pm SD & Total & & & & & \\
\hline Al-Habbo, 2012 & $84.4 \pm 8.7$ & 45 & $100.7 \pm 18.7$ & 45 & $1.9 \%$ & $-16.30(-22.33--10.27)$ & & & \\
\hline Ali, 2009 & $93.4 \pm 9.31$ & 60 & $130.13 \pm 12.84$ & 30 & $2.3 \%$ & $-36.73(-41.89--31.57)$ & & & \\
\hline Ari, 2002 & $103 \pm 14.7$ & 25 & $103.4 \pm 10.1$ & 10 & $1.2 \%$ & $-0.40(-8.91-8.11)$ & - & & \\
\hline Benbassat, 2001 & $104.2 \pm 16$ & 12 & $102 \pm 14.1$ & 15 & $0.8 \%$ & $2.20(-9.33-13.73)$ & & & \\
\hline Boulbou, 2003 & $96.9 \pm 12.8$ & 49 & $103.97 \pm 14.28$ & 22 & $1.6 \%$ & $-7.07(-14.03--0.11)$ & & & \\
\hline Buchmann, 2016 & $96.1 \pm 16.5$ & 91 & $99.6 \pm 18$ & 506 & $2.9 \%$ & $-3.50(-7.24-0.24)$ & $\rightarrow$ & & \\
\hline Ceylan, 2011 & $96.3 \pm 11.4$ & 37 & $92.4 \pm 9.2$ & 25 & $2.3 \%$ & $-6.10(-11.25--0.95)$ & $=$ & & \\
\hline Chance, 2008 & $94.8 \pm 14.4$ & 69 & $98 \pm 1$ & 45 & $3.1 \%$ & $-3.20(-6.61-0.21)$ & $\rightarrow$ & & \\
\hline Dharwadkar, 2011 & $67.44 \pm 16.51$ & 40 & $90.94 \pm 8.19$ & 40 & $2.0 \%$ & $-23.50(-29.21--17.79)$ & & & \\
\hline Guazzi, 2011 & $95.1 \pm 12.2$ & 15 & $97.5 \pm 10.7$ & 15 & $1.3 \%$ & $-2.40(-10.61-5.81)$ & $\rightarrow$ & & \\
\hline Huang, 2014 & $89.14 \pm 17.65$ & 292 & $93.65 \pm 13.34$ & 292 & $3.6 \%$ & $-4.51(-7.05--1.97)$ & $\rightarrow$ & & \\
\hline Isotani, 1999 & $85.3 \pm 7$ & 54 & $87.3 \pm 6.8$ & 34 & $3.4 \%$ & $-2.00(-4.95-0.95)$ & - & & \\
\hline Kabitz, 2008 & $103.2 \pm 10.6$ & 21 & $102.9 \pm 12$ & 23 & $1.7 \%$ & $0.30(-6.38-6.98)$ & - & & \\
\hline Katoh, 1996 & $85.5 \pm 5.4$ & 19 & $88.2 \pm 4.7$ & 16 & $3.1 \%$ & $-2.70(-6.05-0.65)$ & $\ldots$ & & \\
\hline Kaur, 2016 & $85.4 \pm 7.3$ & 50 & $95.26 \pm 16.14$ & 50 & $2.4 \%$ & $-9.86(-14.77--4.95)$ & - & & \\
\hline Khafaie, 2017 & $85.87 \pm 14.06$ & 347 & $86.35 \pm 14.92$ & 386 & $3.8 \%$ & $-0.48(-2.58-1.62)$ & & & \\
\hline Kim, 2011 & $89 \pm 12$ & 2745 & $92 \pm 11$ & 21937 & $4.3 \%$ & $-3.00(-3.47--2.53)$ & - & & \\
\hline Kim, 2017 & $89.3 \pm 0.4$ & 1431 & $92.4 \pm 0.2$ & 7353 & $4.4 \%$ & $-3.10(-3.12--3.08)$ & - & & \\
\hline Klein, 2011 & $75.3 \pm 0.7$ & 76 & $82.6 \pm 0.4$ & 210 & $4.4 \%$ & $-7.30(-7.47--7.13)$ & . & & \\
\hline Kumar, 2016 & $68.825 \pm 15.11$ & 140 & $78.23 \pm 10.63$ & 22 & $1.8 \%$ & $-9.41(-15.86--2.95)$ & - & & \\
\hline Lara Rodriguez, 1995 & $95 \pm 11.53$ & 12 & $94 \pm 12.09$ & 9 & $0.9 \%$ & $-1.00(-9.24-11.24)$ & 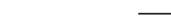 & & \\
\hline Lau, 2004 & $96.7 \pm 12.4$ & 40 & $103.7 \pm 15.2$ & 20 & $1.4 \%$ & $-7.00(-14.69-0.69)$ & & & \\
\hline Lecube, 2010 & $88.4 \pm 19.7$ & 25 & $100.1 \pm 12.4$ & 50 & $1.2 \%$ & $-11.70(-20.15--3.25)$ & & & \\
\hline Lopez-Cano, 2017 & $82.9 \pm 25.9$ & 49 & $101.1 \pm 13.1$ & 98 & $1.4 \%$ & $-18.20(-25.90--10.50)$ & & & \\
\hline Maiolo, 2002 & $98.88 \pm 14.17$ & 12 & $116.52 \pm 13.28$ & 12 & $0.8 \%$ & $-17.64(-28.63--6.65)$ & - & & \\
\hline Matsubara, 1991 & $79.8 \pm 6.5$ & 52 & $80.7 \pm 5.6$ & 48 & $3.7 \%$ & $-0.90(-3.27-1.47)$ & & & \\
\hline Nandhini, 2012 & $67.64 \pm 21.99$ & 45 & $94.4 \pm 27.36$ & 47 & $1.0 \%$ & $-26.76(-36.88--16.64)$ & & & \\
\hline Okur, 2018 & $96.78 \pm 14.43$ & 58 & $99.73 \pm 15.8$ & 52 & $2.1 \%$ & $-2.95(-8.63-2.73)$ & -1 & & \\
\hline Ortiz-Aguirre, 2006 & $86.9 \pm 1.3$ & 144 & $92 \pm 1.3$ & 139 & $4.4 \%$ & $-5.10(-5.40--4.80)$ & - & & \\
\hline Röhling, 2018 & $77.3 \pm 15.6$ & 34 & $94.8 \pm 12.6$ & 26 & $1.6 \%$ & $-17.50(-24.64--10.36)$ & & & \\
\hline Shafiee, 2013 & $107.07 \pm 14.91$ & 180 & $109.82 \pm 15.33$ & 40 & $2.0 \%$ & $-2.75(-8.52-3.02)$ & & & \\
\hline Shah, 2013 & $78.98 \pm 14.09$ & 60 & $88.03 \pm 6.69$ & 60 & $2.8 \%$ & $-9.05(-13.00--5.10)$ & - & & \\
\hline Sinha, 2004 & $80.47 \pm 19.2$ & 29 & $83.5 \pm 14$ & 11 & $0.9 \%$ & $-3.03(-13.86-7.80)$ & & & \\
\hline Tai, 2017 & $77.03 \pm 3.79$ & 63 & $78.59 \pm 2.21$ & 32 & $4.2 \%$ & $-1.56(-2.77--0.35)$ & & & \\
\hline Tayarami, 2018 & $82.3 \pm 15.2$ & 50 & $90.4 \pm 9.4$ & 50 & $2.4 \%$ & $-8.10(-13.05--3.15)$ & - & & \\
\hline Uz-Zaman, 2014 & $81.31 \pm 3.86$ & 60 & $97.5 \pm 9.59$ & 60 & $3.5 \%$ & $-16.19(-18.81--13.57)$ & $\Rightarrow$ & & \\
\hline Weisbrod, 2005 & $90.2 \pm 5.6$ & 8 & $92.2 \pm 5.8$ & 7 & $2.0 \%$ & $-2.00(-7.79-3.79)$ & & & \\
\hline Wilms, 2017 & $84.2 \pm 12.6$ & 65 & $86.7 \pm 13.1$ & 65 & $2.6 \%$ & $-2.50(-6.92-1.92)$ & & & \\
\hline Yeh, 2008 & $92.5 \pm 14.1$ & 1100 & $96.4 \pm 14.6$ & 10162 & $4.3 \%$ & $-3.90(-4.78--3.02)$ & $=$ & & \\
\hline Zamarron, 2001 & $62.7 \pm 29.3$ & 31 & $102.4 \pm 12.8$ & 16 & $0.7 \%$ & $-39.70(-51.77--27.63)-$ & & & \\
\hline Zineldin, 2015 & $73.42 \pm 3.77$ & 45 & $87.02 \pm 5.59$ & 45 & $3.9 \%$ & $-13.60(-51.57--11.63)$ & $\rightarrow$ & & \\
\hline Total (95\% CI) & & 7580 & & 42125 & $100.0 \%$ & $-7.15(-8.27--6.03)$ & 1 & & \\
\hline \multirow{2}{*}{\multicolumn{5}{|c|}{$\begin{array}{l}\text { Heterogeneity: } \tau^{2}=7.42 ; \chi^{2}=3142.85, d f=40(p<0.00001) ; I^{2}=99 \% \\
\text { Test for overall effect: } Z=12.54(p<0.00001)\end{array}$}} & & & -25 & 25 & 30 \\
\hline & & & & & & & Type-2 diabetes & Control & \\
\hline
\end{tabular}

b)

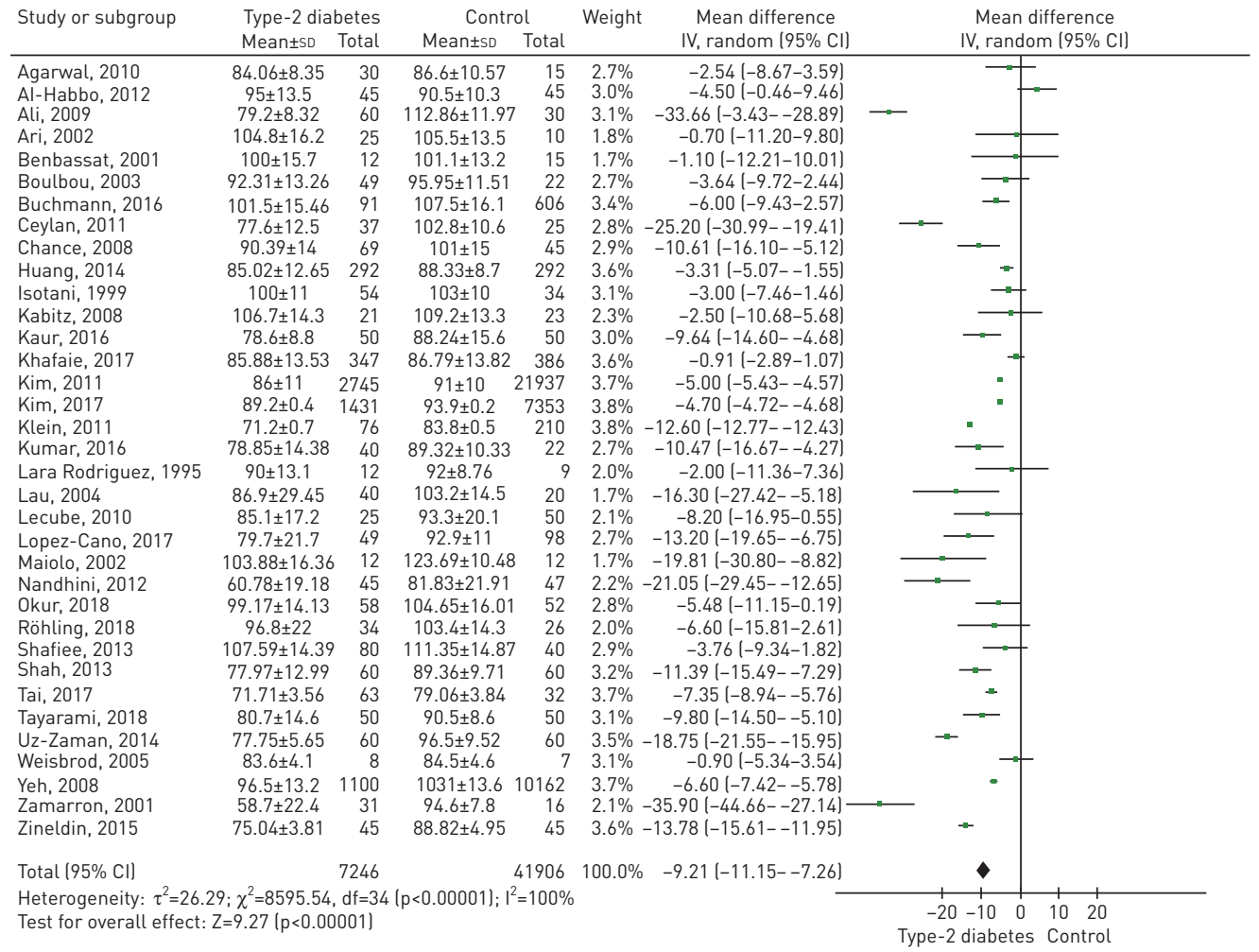

FIGURE 2 Forest plots of (a) \% predicted forced expiratory volume in $1 \mathrm{~s}$ and (b) \% predicted forced vital capacity. 


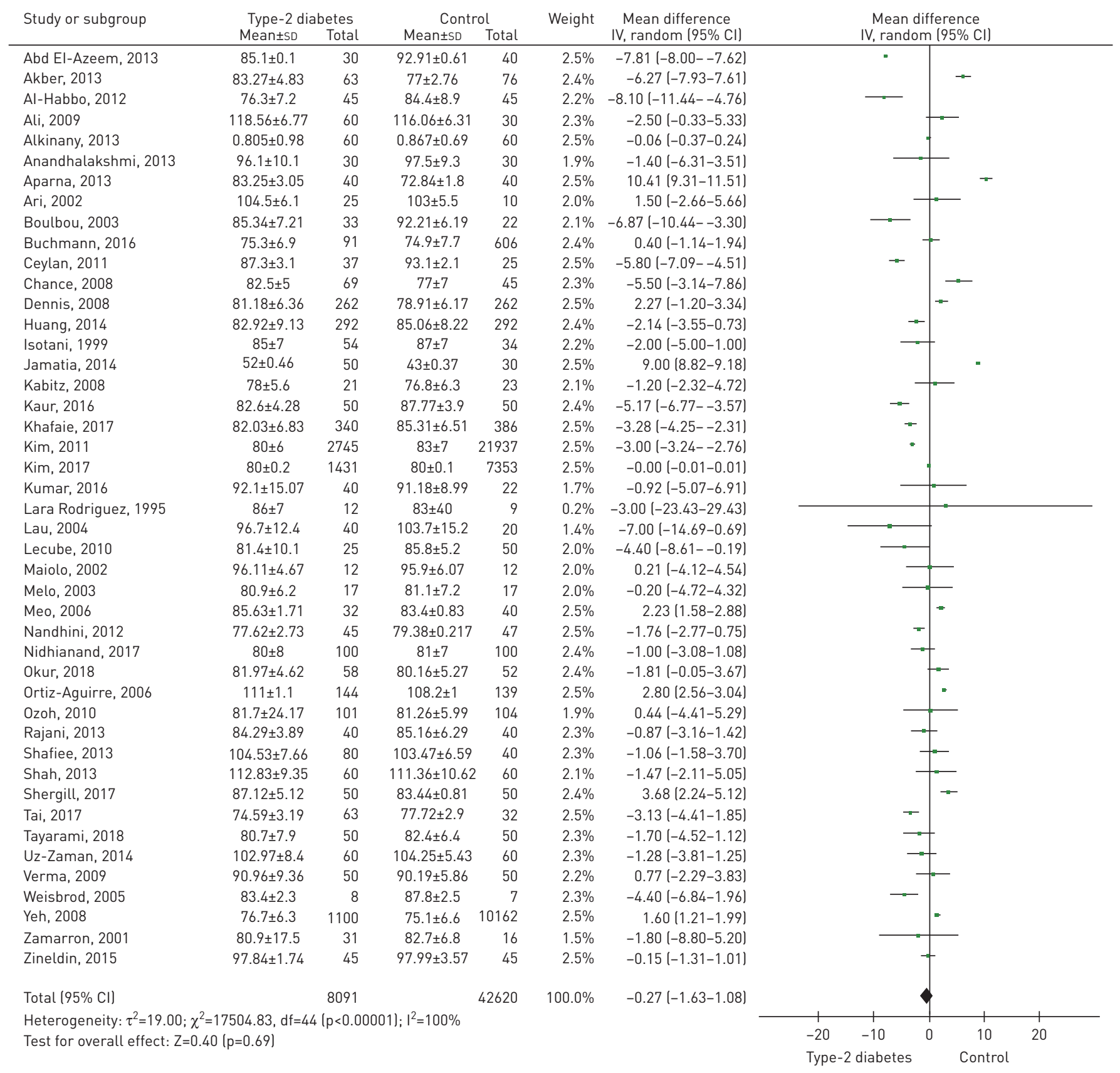

FIGURE 3 Forest plot of forced expiratory volume in $1 \mathrm{~s} /$ forced vital capacity ratio (\%).

$\mathrm{FEF}_{25-75 \%}$

A total of 13 studies included data on $\% \mathrm{FEF}_{25-75 \%}$, and 12 included data on $\mathrm{FEF}_{25-75 \%}\left(\mathrm{~L} \cdot \mathrm{s}^{-1}\right)$. Figure $4 \mathrm{a}$ and figure S3 (supplementary material) show the forest plots of the effect estimates. For the patients with T2D, the pooled estimates for $\% \mathrm{FEF}_{25-75 \%}$ and $\mathrm{FEF}_{25-75 \%}\left(\mathrm{~L} \cdot \mathrm{s}^{-1}\right)$ were -9.89 (95\% CI -14.42 to -5.36 ; $\mathrm{p}<0.0001)$ and -0.48 ( $95 \%$ CI -0.71 to -0.24 ; $\mathrm{p}<0.0001)$, respectively.

PEF

A total of 15 studies included data on \% PEF, and 19 included data on PEF $\left(\mathrm{L} \cdot \mathrm{s}^{-1}\right)$. Figure $4 \mathrm{~b}$ and figure S4 (supplementary material) show the comparison forest plot. The pooled effect estimates for the patients with T2D were -9.79 (95\% CI -13.42 to -6.15 ; $\mathrm{p}<0.0001)$ for $\% \mathrm{PEF}$ and -1.07 (95\% CI -1.43 to -0.71 ; $\mathrm{p}<0.0001)$ for $\mathrm{PEF}\left(\mathrm{L} \cdot \mathrm{s}^{-1}\right)$. 
a)

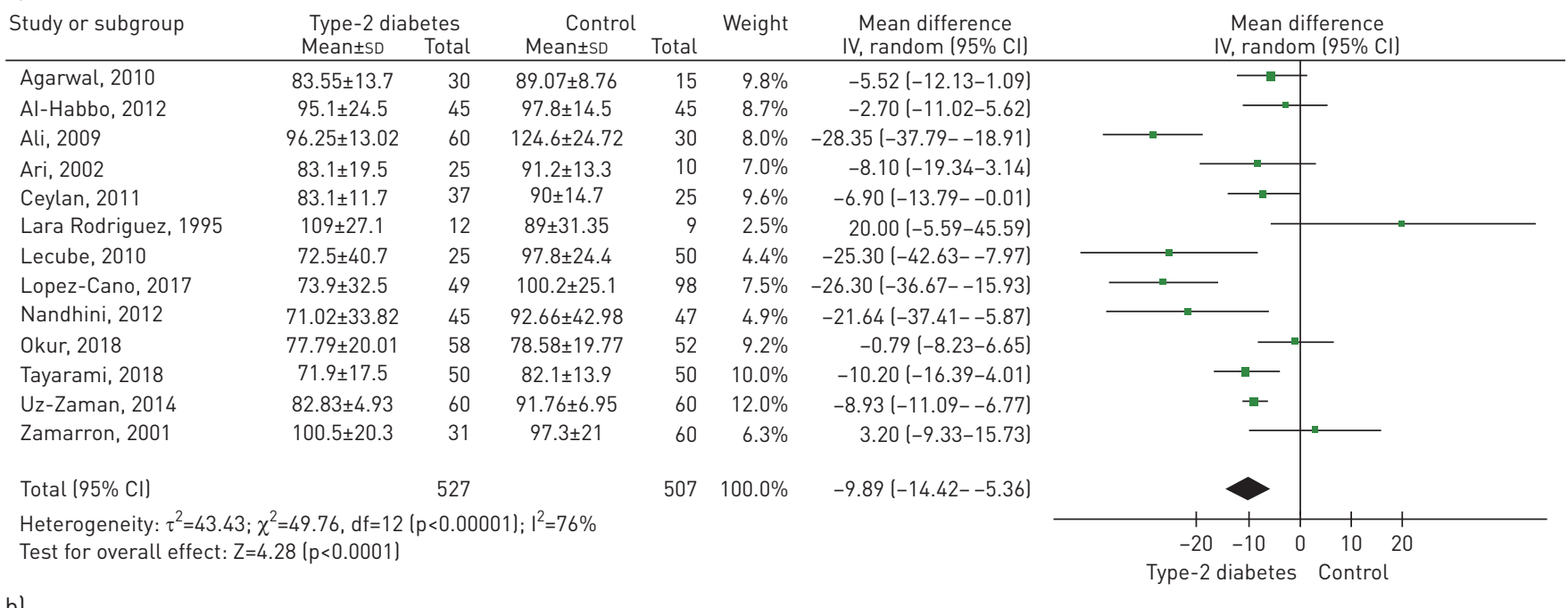

b)

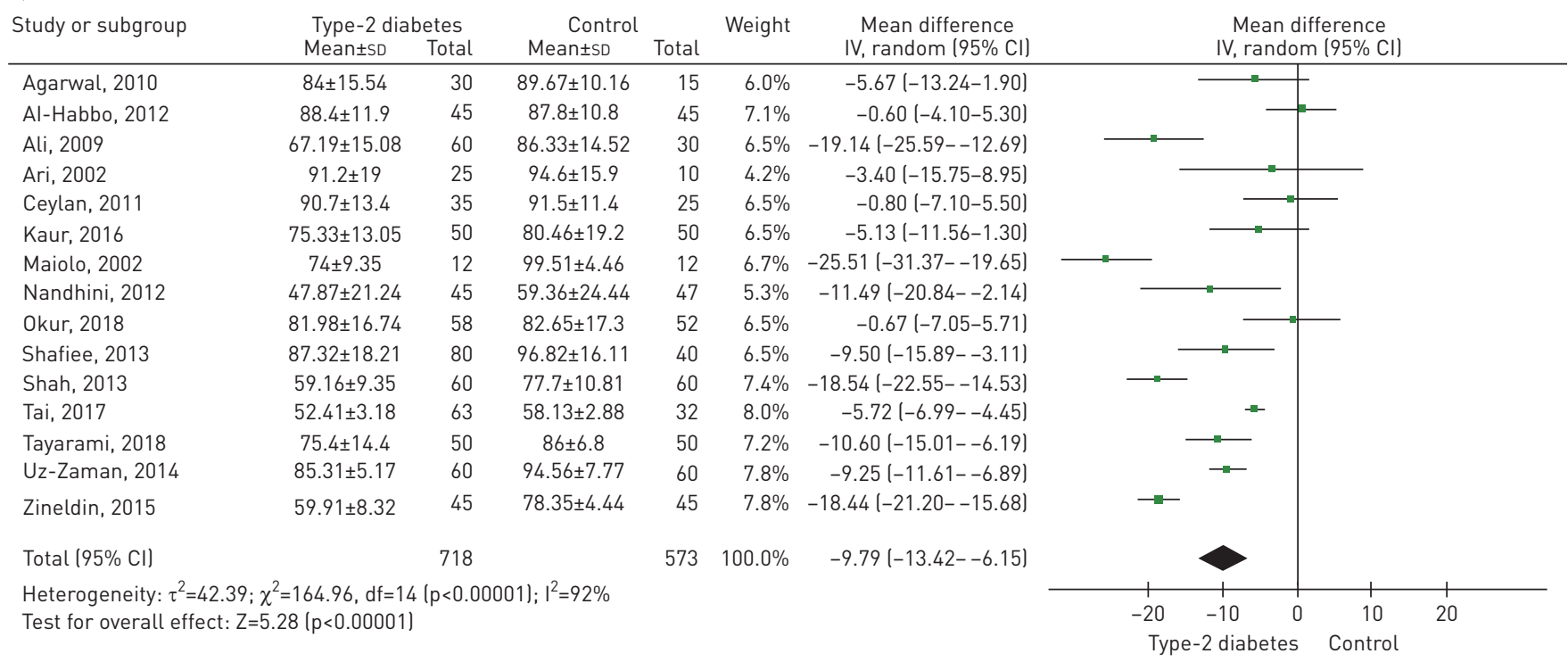

c)

\begin{tabular}{|c|c|c|c|c|c|c|c|c|c|}
\hline \multirow[t]{2}{*}{ Study or subgroup } & \multicolumn{2}{|c|}{ Type-2 diabetes } & \multicolumn{2}{|l|}{ Control } & \multirow[t]{2}{*}{ Weight } & \multirow{2}{*}{$\begin{array}{l}\text { Mean difference } \\
\text { IV, random }(95 \% \mathrm{CI})\end{array}$} & \multirow{2}{*}{\multicolumn{3}{|c|}{$\begin{array}{l}\text { Mean difference } \\
\text { IV, random }(95 \% \mathrm{CI})\end{array}$}} \\
\hline & Mean \pm SD & Total & Mean \pm SD & Total & & & & & \\
\hline Agarwal, 2010 & $77.51 \pm 15.44$ & 30 & $84.53 \pm 13.9$ & 15 & $7.1 \%$ & $-7.03(-15.97-1.91)$ & & - & \\
\hline Benbassat, 2001 & $113.6 \pm 24$ & 12 & $107.3 \pm 16$ & 15 & $3.6 \%$ & $6.30(-9.51-22.11)$ & & & \\
\hline Boulbou, 2003 & $90.51 \pm 22.17$ & 33 & $106.43 \pm 15.66$ & 22 & $6.3 \%$ & $-15.92(-25.92--5.92)$ & & & \\
\hline Buyukhatipoglu, 2011 & $119 \pm 21$ & 80 & $122 \pm 19$ & 40 & $8.2 \%$ & $-3.00(-10.47-4.47)$ & & & \\
\hline Ceylan, 2011 & $90.3 \pm 6.1$ & 37 & $96.3 \pm 6.8$ & 25 & $11.8 \%$ & $-6.00(-9.31--2.69)$ & - & & \\
\hline Guazzi, 2011 & $87.2 \pm 5.4$ & 15 & $101.3 \pm 7.1$ & 15 & $10.8 \%$ & $-14.10(-18.61--9.59)$ & & & \\
\hline Kumar, 2016 & $71.6 \pm 14.795$ & 40 & $76.77 \pm 19.25$ & 22 & $6.8 \%$ & $-5.17(-14.43-4.09)$ & & & \\
\hline Ozsahin, 2006 & $92.2 \pm 18.5$ & 25 & $102.6 \pm 17.8$ & 15 & $5.4 \%$ & $-10.40(-21.96-1.16)$ & & & \\
\hline Saler, 2009 & $93.6 \pm 22.2$ & 68 & $103.3 \pm 25.4$ & 80 & $8.0 \%$ & $-9.70(-17.37--2.03)$ & - & & \\
\hline Tai, 2017 & $85.03 \pm 2.66$ & 63 & $87.22 \pm 2.74$ & 32 & $13.0 \%$ & $-2.19(-3.34--1.04)$ & - & & \\
\hline Uz-Zaman, 2014 & $94.35 \pm 18.63$ & 60 & $108.53 \pm 13.13$ & 60 & $9.7 \%$ & $-14.18(-19.95--8.41)$ & & & \\
\hline Zamarron, 2001 & $95.2 \pm 6.7$ & 31 & $95.5 \pm 11.5$ & 16 & $9.4 \%$ & $-0.30(-6.41-5.81)$ & & & \\
\hline Total $(95 \% \mathrm{CI})$ & & 494 & & 357 & $100.0 \%$ & $-7.13(-10.62--3.64)$ & & & \\
\hline \multicolumn{7}{|c|}{$\begin{array}{l}\text { Heterogeneity: } \tau^{2}=24.09 ; \chi^{2}=54.23, d f=11(p<0.00001) ; I^{2}=80 \% \\
\text { Test for overall effect: } Z=4.01(p<0.0001)\end{array}$} & -20 & 10 & 20 \\
\hline
\end{tabular}

FIGURE 4 Forest plots of (a) \% predicted forced expiratory flow between $25 \%$ and $75 \%$ of total lung capacity, (b) \% predicted peak expiratory flow, and (c) \% predicted diffusion capacity of the lungs for carbon monoxide. 
$D_{L C O}$

A total of 12 studies included data on $\% \mathrm{DL}_{\mathrm{CO}}$, and ten included data on $D_{\mathrm{LCO}}\left(\mathrm{mL} \cdot \mathrm{min}^{-1} \cdot \mathrm{mmHg}^{-1}\right)$. Figure 4c and figure S5 (supplementary material) show the comparison forest plot. The pooled effect estimates for the patients with T2D were -7.13 (95\% CI -10.62 to $-3.64 ; \mathrm{p}<0.0001)$ for $\% D_{\mathrm{LCO}}$ and $-3.42(95 \% \mathrm{CI}-5.14$ to $-1.70 ; \mathrm{p}<0.0001)$ for $D_{\mathrm{LCO}}\left(\mathrm{mL} \cdot \mathrm{min}^{-1} \cdot \mathrm{mmHg}^{-1}\right)$.

There was significant heterogeneity for all parameters of the pulmonary function tests $\left(\mathrm{I}^{2}, 80-100 \%\right)$.

\section{Subgroup analysis}

Table 2 and table S1 (supplementary material) present the meta-regression analysis pre-specified by subgroup.

Sex

Fifteen studies reported data differentiated by sex. A comparison could be established for \% FEV $1, \mathrm{FEV}_{1}$ (L), \% FVC, FVC (L), FEV $/$ FVC ratio and PEF $\left(\mathrm{L} \cdot \mathrm{s}^{-1}\right)$. There were no differences by sex $(\mathrm{p}>0.25$ for all cases).

\section{Tobacco use}

Fourteen studies included patients who smoked and those who did not, and 43 studies included exclusively nonsmokers. Another nine studies did not report data on tobacco use. There was heterogeneity between the groups; the effect estimate for the patients with T2D who did not smoke presented a reduction in $\% \mathrm{FEV}_{1}, \% \mathrm{FVC}$, FVC $(\mathrm{L})(\mathrm{p} \leqslant 0.01$ for all $)$ and PEF $\left(\mathrm{L} \cdot \mathrm{s}^{-1}\right)(\mathrm{p}<0.001)$, which was higher than in the other studies that included smokers and nonsmokers.

\section{Geographical region}

The same abnormal pulmonary function test results were observed in the patients with T2D in all continents. However, we observed heterogeneity between the various continents in $\% \mathrm{FEV}_{1}, \% \mathrm{FVC}, \mathrm{FEV}_{1} /$ FVC ratio, $\operatorname{FEF}_{25-75 \%}\left(\mathrm{~L} \cdot \mathrm{s}^{-1}\right), \% \mathrm{PEF}, D_{\mathrm{LCO}}\left(\mathrm{mL} \cdot \mathrm{min}^{-1} \cdot \mathrm{mmHg}^{-1}\right)($ all $\mathrm{p}<0.001)$ and PEF $\left(\mathrm{L} \cdot \mathrm{s}^{-1}\right)(\mathrm{p}=0.004)$.

\section{Sensitivity analysis}

When we applied the fixed-effects model, we observed the same abnormal pulmonary function test results. The same result occurred when we performed an analysis separated by publication year, size of the T2D group, study quality and even when we included the articles from predatory journals and from the proceedings of congresses (table 3 and table S2 supplementary material). The magnitude of the effect estimates was higher for $\% \mathrm{FEV}_{1}, \% \mathrm{FVC}, \% \mathrm{FEF}_{25-75 \%} \% \mathrm{PEF}$ and $\% D_{\mathrm{LCO}}$ when only good quality studies were included in the meta-analysis. The removal of the study with greatest weight in each pulmonary function test did not change the results.

\section{Publication bias}

The funnel plots showed asymmetry, indicating the presence of potential publication biases (figure 5 and figure S6 supplementary material).

\section{Discussion}

The results of our meta-analysis show that all of the pulmonary function test results, except the $\mathrm{FEV}_{1} / \mathrm{FVC}$ ratio, were decreased for the patients with T2D. This pulmonary function impairment in $\mathrm{T} 2 \mathrm{D}$ is observed worldwide, also in nonsmokers and is independent of sex.

Various qualitative reviews have been published on the influence of diabetes on pulmonary function [75-80], all of which have reported the presence of a reduction in $\mathrm{FEV}_{1}$ and FVC in patients with diabetes. In 2010, VAN DEN BORST et al. [7] published a meta-analysis on pulmonary function in patients with diabetes, which included 16 studies with 1695 patients with T2D and 10260 controls. The pooled difference in the $\% \mathrm{FEV}_{1}, \% \mathrm{FVC}$ and $\% D_{\mathrm{LCO}}$ was $-4.86,-6.67$ and -9.30 , respectively, with no difference in the $\mathrm{FEV}_{1} / \mathrm{FVC}$ ratio. Their results are consistent with those observed in our meta-analysis.

Recently, SAINI et al. [8] reported another meta-analysis with 22 studies that included 7526 patients with T2D and 43641 controls. The pooled difference in the \% FEV 1 and \% FVC was -6.37 and -6.56 respectively, with no difference in the $\mathrm{FEV}_{1} / \mathrm{FVC}$ ratio. The meta-analysis also presented data on $\mathrm{FEV}_{1}(\mathrm{~L})$, FVC (L), with differences of -0.27 and $-0.31 \mathrm{~L}$, respectively, which were consistent with those observed in our meta-analysis. However, our meta-analysis and that of SAINI et al. [8] differ in the included studies. In our meta-analysis there are 20 studies that SAINI et al. [8] did not include [38, 40, 43, 44, 47-50, 53, 57, 59-68]. Moreover, we did not include nine of the studies in the SAINI et al. [8] meta-analysis because we considered that the studies did not clearly state that they only included patients with T2D [supplementary 
TABLE 2 Meta-regression with subgroup analysis

\begin{tabular}{|c|c|c|c|c|c|c|c|c|c|c|c|c|c|c|c|}
\hline & \multicolumn{5}{|c|}{$\% \mathrm{FEV}_{1}$} & \multicolumn{5}{|c|}{$\%$ FVC } & \multicolumn{5}{|c|}{ FEV $_{1} /$ FVC $(\%)$} \\
\hline & Studies & Participants & $\begin{array}{c}\text { Effect } \\
\text { estimate }\end{array}$ & $I^{2}$ & $\mathrm{p}$ & Studies & Participants & $\begin{array}{c}\text { Effect } \\
\text { estimate }\end{array}$ & $I^{2}$ & p & Studies & Participants & $\begin{array}{c}\text { Effect } \\
\text { estimate }\end{array}$ & $I^{2}$ & $\mathrm{p}$ \\
\hline Male & 5 & 667 & $\begin{array}{l}-13.10 \\
(-22.57 \\
-3.64)\end{array}$ & $96 \%$ & 0.007 & 5 & 667 & $\begin{array}{c}-13.71 \\
(-22.02 \\
-5.39)\end{array}$ & $95 \%$ & 0.001 & 11 & 1099 & $\begin{array}{c}2.69(0.70 \\
4.67)\end{array}$ & $92 \%$ & 0.008 \\
\hline Female & 3 & 473 & $\begin{array}{c}-9.00 \\
(-19.86,1.85)\end{array}$ & $82 \%$ & 0.10 & 3 & 473 & $\begin{array}{c}-9.58 \\
(-18.07 \\
-1.09]\end{array}$ & $70 \%$ & 0.03 & 7 & 737 & $\begin{array}{c}2.84(-1.89 \\
7.57)\end{array}$ & $96 \%$ & 0.24 \\
\hline Nonsmokers & 25 & 2357 & $\begin{array}{c}-9.67 \\
(-13.05 \\
-6.29)\end{array}$ & $94 \%$ & $<0.001$ & 23 & 2252 & $\begin{array}{c}-10.84 \\
(-14.12 \\
-7.57)\end{array}$ & $93 \%$ & $<0.001$ & 32 & 3257 & $\begin{array}{c}-0.21 \\
(-3.35,2.93)\end{array}$ & $100 \%$ & 0.90 \\
\hline \multicolumn{16}{|l|}{ Continent } \\
\hline Africa & 1 & 90 & NA & NA & NA & 1 & 90 & NA & NA & NA & 3 & 365 & $\begin{array}{c}-2.70 \\
(-9.01,3.62)\end{array}$ & $99 \%$ & 0.40 \\
\hline America & 5 & 11966 & $\begin{array}{c}-4.99(-6.72 \\
-3.26)\end{array}$ & $98 \%$ & $<0.001$ & 4 & 11683 & $\begin{array}{c}-8.77 \\
(-13.43 \\
-4.11)\end{array}$ & $99 \%$ & $<0.001$ & 6 & 12238 & $\begin{array}{c}2.48(1.56 \\
3.40)\end{array}$ & $85 \%$ & $<0.001$ \\
\hline Asia & 21 & 36202 & $\begin{array}{c}-7.50(-9.01 \\
-6.00)\end{array}$ & $95 \%$ & $<0.001$ & 18 & 35992 & $\begin{array}{c}-7.91(-9.40 \\
-6.43)\end{array}$ & $95 \%$ & $<0.001$ & 26 & 36944 & $\begin{array}{c}0.02(-1.80 \\
1.83)\end{array}$ & $100 \%$ & 0.99 \\
\hline Europe & 13 & 1432 & $\begin{array}{c}-8.93 \\
(-13.25 \\
-4.62)\end{array}$ & $82 \%$ & $<0.001$ & 11 & 1372 & $\begin{array}{r}-11.48 \\
(-17.38 \\
-5.57)\end{array}$ & $88 \%$ & $<0.001$ & 9 & 1149 & $\begin{array}{c}-1.53 \\
(-4.21,1.16)\end{array}$ & $89 \%$ & 0.26 \\
\hline $\begin{array}{c}\text { Oceania } \\
\text { BMI }\left(\mathbf{k g} \cdot \mathbf{m}^{-2}\right)\end{array}$ & 1 & 15 & NA & NA & 0.50 & 1 & 15 & NA & NA & NA & 1 & 15 & NA & NA & NA \\
\hline$<25$ & 9 & 1809 & $\begin{array}{c}-10.95 \\
(-16.58 \\
-5.33)\end{array}$ & $96 \%$ & $<0.001$ & 5 & 927 & $\begin{array}{c}-12.26 \\
(-23.01 \\
-1.51)\end{array}$ & $98 \%$ & 0.03 & 7 & 1152 & $\begin{array}{c}1.41(-3.31 \\
6.14)\end{array}$ & $99 \%$ & 0.56 \\
\hline $25-29.9$ & 14 & 35064 & $\begin{array}{c}-3.46(-5.54 \\
-2.38)\end{array}$ & $94 \%$ & $<0.001$ & 14 & 35515 & $\begin{array}{c}-5.78(-7.03 \\
-4.53)\end{array}$ & $90 \%$ & $<0.001$ & 16 & 36003 & $\begin{array}{c}-0.28 \\
(-1.61,1.05)\end{array}$ & $99 \%$ & 0.68 \\
\hline $30-39.9$ & 6 & 11856 & $\begin{array}{c}-6.50(-9.14 \\
-3.86)\end{array}$ & $93 \%$ & $<0.001$ & 6 & 11856 & $\begin{array}{c}-9.66 \\
(-13.67 \\
-5.65)\end{array}$ & $98 \%$ & $<0.001$ & 5 & 11649 & $\begin{array}{c}3.25 \text { (0.83 } \\
5.68)\end{array}$ & $92 \%$ & 0.009 \\
\hline$\geqslant 40$ & 3 & 352 & $\begin{array}{r}-10.40 \\
(-20.45 \\
-0.35)\end{array}$ & $85 \%$ & 0.04 & 2 & 222 & $\begin{array}{r}-11.44 \\
(-16.63 \\
-6.24)\end{array}$ & $0 \%$ & $<0.001$ & 1 & 75 & NA & NA & NA \\
\hline
\end{tabular}


TABLE 2 Continued

\begin{tabular}{|c|c|c|c|c|c|c|c|c|c|c|c|c|c|c|c|}
\hline & \multicolumn{5}{|c|}{$\% \mathrm{FEF}_{25-75}$} & \multicolumn{5}{|c|}{$\%$ PEF } & \multicolumn{5}{|c|}{$\% D_{\text {LCo }}$} \\
\hline & Studies & Participants & $\begin{array}{c}\text { Effect } \\
\text { estimate }\end{array}$ & $I^{2}$ & $p$ & Studies & Participants & $\begin{array}{c}\text { Effect } \\
\text { estimate }\end{array}$ & $I^{2}$ & p & Studies & Participants & $\begin{array}{c}\text { Effect } \\
\text { estimate }\end{array}$ & $I^{2}$ & p \\
\hline Male & 2 & 210 & $\begin{array}{c}-17.18 \\
(-138.25 \\
3.89)\end{array}$ & $94 \%$ & 0.11 & 3 & 300 & $\begin{array}{l}-18.55 \\
(-20.69 \\
-16.40)\end{array}$ & $0 \%$ & $<0.001$ & 0 & NA & NA & NA & NA \\
\hline Female & 1 & 75 & $\begin{array}{c}-25.30 \\
(-42.63 \\
-7.97)\end{array}$ & NA & NA & 1 & 24 & $\begin{array}{l}-25.51 \\
(-31.37 \\
-19.65)\end{array}$ & NA & NA & 0 & NA & NA & NA & NA \\
\hline Nonsmokers & 10 & 742 & $\begin{array}{r}-11.04 \\
(-16.29 \\
-5.78)\end{array}$ & $77 \%$ & $<0.001$ & 10 & 844 & $\begin{array}{r}-11.03 \\
(-15.25 \\
-6.82)\end{array}$ & $92 \%$ & $<0.001$ & 12 & 851 & $\begin{array}{c}-7.13 \\
(-10.62 \\
-3.64)\end{array}$ & $80 \%$ & $<0.001$ \\
\hline \multicolumn{16}{|l|}{ Continent } \\
\hline Africa & 0 & 90 & NA & NA & NA & 1 & 90 & NA & NA & NA & 0 & 0 & NA & NA & NA \\
\hline America & 1 & 21 & NA & NA & NA & 0 & 0 & NA & NA & NA & 0 & 0 & NA & NA & NA \\
\hline Asia & 6 & 537 & $\begin{array}{l}-11.44 \\
(-17.07 \\
-5.81)\end{array}$ & $77 \%$ & $<0.001$ & 10 & 972 & $\begin{array}{c}-9.34 \\
(-12.75 \\
-5.93)\end{array}$ & $86 \%$ & $<0.001$ & 5 & 349 & $\begin{array}{c}-5.60 \\
(-11.83 \\
-0.62)\end{array}$ & $78 \%$ & 0.08 \\
\hline Europe & 6 & 476 & $\begin{array}{c}-9.88(-18.65 \\
-1.11)\end{array}$ & $78 \%$ & 0.03 & 4 & 229 & $\begin{array}{c}-7.78 \\
(-21.43,5.88)\end{array}$ & $93 \%$ & 0.26 & 7 & 502 & $\begin{array}{c}-8.07 \\
(-12.34 \\
-3.79)\end{array}$ & $68 \%$ & $<0.001$ \\
\hline $\begin{array}{l}\text { Oceania } \\
\text { BMI }\left(\mathbf{k g} \cdot \mathbf{m}^{-2} \text { ) }\right.\end{array}$ & 0 & 0 & NA & NA & NA & 0 & 0 & NA & NA & NA & 0 & 0 & NA & NA & NA \\
\hline$<25$ & 3 & 255 & $\begin{array}{l}-13.36 \\
(-23.15 \\
-3.57)\end{array}$ & $88 \%$ & 0.007 & 4 & 345 & $\begin{array}{r}-13.32 \\
(-19.69 \\
-6.96)\end{array}$ & $91 \%$ & $<0.001$ & 3 & 205 & $\begin{array}{r}-11.83 \\
(-16.30 \\
-7.36)\end{array}$ & $0 \%$ & $<0.001$ \\
\hline $25-29.9$ & 1 & 35 & NA & NA & NA & 3 & 250 & $\begin{array}{c}-5.84(-7.08 \\
-4.60)\end{array}$ & $0 \%$ & $<0.001$ & 6 & 507 & $\begin{array}{c}-5.20(-9.72 \\
-0.68)\end{array}$ & $59 \%$ & 0.002 \\
\hline $30-39.9$ & 1 & 110 & NA & NA & NA & 2 & 134 & $\begin{array}{c}-13.12 \\
(-37.47 \\
11.22)\end{array}$ & $97 \%$ & 0.29 & 0 & 0 & NA & NA & NA \\
\hline$\geqslant 40$ & 2 & 222 & $\begin{array}{l}-26.04 \\
(-34.93 \\
-17.14)\end{array}$ & $0 \%$ & $<0.001$ & 0 & 0 & NA & NA & NA & 0 & 0 & NA & NA & NA \\
\hline
\end{tabular}




\begin{tabular}{|c|c|c|c|c|c|c|c|c|c|c|c|c|}
\hline & \multicolumn{4}{|c|}{$\% \mathrm{FEV}_{1}$} & \multicolumn{4}{|c|}{$\%$ FVC } & \multicolumn{4}{|c|}{$\mathrm{FEV}_{1} / \mathrm{FVC}(\%)$} \\
\hline & Studies & Participants & Effect estimate & $I^{2}$ & Studies & Participants & Effect estimate & $I^{2}$ & Studies & Participants & $\begin{array}{c}\text { Effect } \\
\text { estimate }\end{array}$ & $1^{2}$ \\
\hline \multicolumn{13}{|l|}{ Statistical analysis method } \\
\hline Random effect & 41 & 49705 & $\begin{array}{c}-7.15(-8.27 \\
-6.03)\end{array}$ & $99 \%$ & 35 & 49152 & $\begin{array}{c}-9.21(-11.15 \\
-7.26)\end{array}$ & $100 \%$ & 45 & 50711 & $\begin{array}{c}-0.27(-1.63 \\
1.08)\end{array}$ & $100 \%$ \\
\hline Fixed effect & & & $\begin{array}{c}-3.18(-3.20 \\
-3.16)\end{array}$ & & & & $\begin{array}{l}-4.82(-4.85 \\
-4.80)\end{array}$ & & & & $\begin{array}{l}0.01(-0.00 \\
0.02)\end{array}$ & \\
\hline \multicolumn{13}{|l|}{ Publication year } \\
\hline Before 2000 & 4 & 244 & $\begin{array}{c}-1.59(-3.19 \\
0.01)\end{array}$ & $0 \%$ & 2 & 109 & $\begin{array}{c}-2.81(-6.84 \\
1.21)\end{array}$ & $0 \%$ & 2 & 109 & $\begin{array}{c}-1.94(-4.92 \\
1.05)\end{array}$ & $0 \%$ \\
\hline 2000-2009 & 14 & 12142 & $\begin{array}{l}-8.14(-10.96 \\
-5.31)\end{array}$ & $93 \%$ & 11 & 11789 & $\begin{array}{c}-11.96(-18.66 \\
-5.26)\end{array}$ & $95 \%$ & 15 & 12759 & $\begin{array}{l}1.16(0.27 \\
2.05)\end{array}$ & $86 \%$ \\
\hline 2010-2019 & 23 & 37319 & $\begin{array}{l}-8.00(-9.48 \\
-6.51)\end{array}$ & $99 \%$ & 22 & 37254 & $\begin{array}{c}-8.67(-11.04 \\
-6.29)\end{array}$ & $100 \%$ & 28 & 37843 & $\begin{array}{c}-0.53(-2.39 \\
1.33)\end{array}$ & $100 \%$ \\
\hline \multicolumn{13}{|l|}{ Type-2 diabetes group size } \\
\hline$<50$ patients & 21 & 1207 & $\begin{array}{c}-10.29(-13.88 \\
-6.70)\end{array}$ & $86 \%$ & 19 & 1155 & $\begin{array}{c}-9.45(-13.67 \\
-5.22)\end{array}$ & $89 \%$ & 20 & 1168 & $\begin{array}{c}-1.69(-4.86 \\
1.48)\end{array}$ & $99 \%$ \\
\hline$\geqslant 50$ patients & 20 & 48498 & $\begin{array}{c}-5.68(-7.01 \\
-4.34)\end{array}$ & $99 \%$ & 16 & 47997 & $\begin{array}{c}-9.16(-11.80 \\
-6.51)\end{array}$ & $100 \%$ & 25 & 49543 & $\begin{array}{c}0.69(-0.93 \\
2.30)\end{array}$ & $100 \%$ \\
\hline \multicolumn{13}{|l|}{ Study quality } \\
\hline Only good quality studies & 32 & 48201 & $\begin{array}{c}-7.95(-9.30 \\
-6.60)\end{array}$ & $99 \%$ & 31 & 48276 & $\begin{array}{c}-9.53(-11.59 \\
-7.47)\end{array}$ & $100 \%$ & 38 & 49359 & $\begin{array}{c}-0.52(-2.13 \\
1.10)\end{array}$ & $100 \%$ \\
\hline $\begin{array}{l}\text { Including predatory journals } \\
\text { and grey literature }\end{array}$ & 57 & 51845 & $\begin{array}{c}-1.27(-1.83 \\
-0.71)\end{array}$ & $100 \%$ & 48 & 50973 & $\begin{array}{c}-1.73(-2.39 \\
-1.08)\end{array}$ & $100 \%$ & 69 & 53875 & $\begin{array}{l}0.13(-0.02 \\
-0.28)\end{array}$ & $96 \%$ \\
\hline \multicolumn{13}{|c|}{ Excluding the highest-weight study } \\
\hline & 40 & 40921 & $\begin{array}{l}-7.37(-8.53 \\
-6.20)\end{array}$ & $96 \%$ & 34 & 40368 & $\begin{array}{c}-9.39(-11.42 \\
-7.36)\end{array}$ & $98 \%$ & 44 & 26029 & $\begin{array}{c}-0.21(-1.66 \\
1.23)\end{array}$ & $100 \%$ \\
\hline
\end{tabular}




\begin{tabular}{|c|c|c|c|c|c|c|c|c|c|c|c|c|}
\hline & \multicolumn{4}{|c|}{$\% \mathrm{FEF}_{25-75 \% 1}$} & \multicolumn{4}{|c|}{$\%$ PEF } & \multicolumn{4}{|c|}{$\% D_{\text {Lco }}$} \\
\hline & Studies & Participants & Effect estimate & $I^{2}$ & Studies & Participants & Effect estimate & $I^{2}$ & Studies & Participants & Effect estimate & $I^{2}$ \\
\hline \multicolumn{13}{|l|}{ Statistical analysis method } \\
\hline Random effect & 13 & 1034 & $\begin{array}{c}-9.89(-14.42 \\
-5.36)\end{array}$ & $76 \%$ & 15 & 1291 & $\begin{array}{c}-9.79(-13.42 \\
-6.15)\end{array}$ & $92 \%$ & 12 & 851 & $\begin{array}{c}-7.13(-10.62 \\
-3.64)\end{array}$ & $93 \%$ \\
\hline Fixed effect & & & $\begin{array}{c}-9.02(-10.68 \\
-7.36)\end{array}$ & & & & $\begin{array}{c}-8.73(-9.62 \\
-7.85)\end{array}$ & & & & $\begin{array}{c}-3.79(-4.78 \\
-2.80)\end{array}$ & \\
\hline \multicolumn{13}{|l|}{ Publication year } \\
\hline Before 2000 & 1 & 21 & NA & NA & 0 & 0 & NA & NA & 0 & 0 & NA & NA \\
\hline $2000-2009$ & 2 & 82 & $\begin{array}{l}-2.80(-13.85 \\
8.25)\end{array}$ & $42 \%$ & 2 & 59 & $\begin{array}{c}-15.15(-36.78 \\
6.47)\end{array}$ & $90 \%$ & 6 & 347 & $\begin{array}{c}-8.26(-14.58 \\
-1.94)\end{array}$ & $72 \%$ \\
\hline $2010-2019$ & 10 & 931 & $\begin{array}{c}-11.85(-16.68 \\
-7.03)\end{array}$ & $78 \%$ & 13 & 1232 & $\begin{array}{c}-8.93(-12.50 \\
-5.36)\end{array}$ & $91 \%$ & 6 & 504 & $\begin{array}{c}-5.99(-9.80 \\
-2.18)\end{array}$ & $76 \%$ \\
\hline \multicolumn{13}{|l|}{ Type 2 diabetes group size } \\
\hline$<50$ patients & 9 & 614 & $\begin{array}{l}-9.01(-15.84 \\
-2.19)\end{array}$ & $72 \%$ & 7 & 436 & $\begin{array}{c}-9.47(-17.73 \\
-1.22)\end{array}$ & $93 \%$ & 8 & 368 & $\begin{array}{c}-7.29(-11.66 \\
-2.93)\end{array}$ & $66 \%$ \\
\hline$\geqslant 50$ patients & 4 & 420 & $\begin{array}{c}-11.37(-18.96 \\
-3.78)\end{array}$ & $86 \%$ & 8 & 855 & $\begin{array}{l}-9.84(-13.56 \\
-6.12)\end{array}$ & $88 \%$ & 4 & 486 & $\begin{array}{c}-7.01(-13.43 \\
-0.58)\end{array}$ & $84 \%$ \\
\hline \multicolumn{13}{|l|}{ Study quality } \\
\hline Only good quality studies & 12 & 1013 & $\begin{array}{c}-10.60(-15.03 \\
-6.17)\end{array}$ & $75 \%$ & 15 & 1291 & $\begin{array}{c}-9.79(-13.42 \\
-6.15)\end{array}$ & $92 \%$ & 10 & 749 & $\begin{array}{c}-7.08(-10.92 \\
-3.25)\end{array}$ & $83 \%$ \\
\hline $\begin{array}{l}\text { Including predatory journals } \\
\text { and grey literature }\end{array}$ & 18 & 1722 & $\begin{array}{c}-0.57(-0.81 \\
-0.32)\end{array}$ & $82 \%$ & 21 & 1961 & $\begin{array}{c}-0.73(-1.01 \\
-0.46)\end{array}$ & $88 \%$ & 13 & 971 & $\begin{array}{c}-0.51(-0.76 \\
-0.26)\end{array}$ & $69 \%$ \\
\hline \multicolumn{13}{|c|}{ Excluding the highest-weight study } \\
\hline & 12 & 914 & $\begin{array}{c}-10.07(-16.01 \\
-4.13)\end{array}$ & $78 \%$ & 14 & 1196 & $\begin{array}{c}-10.10(-14.20 \\
-6.01)\end{array}$ & $89 \%$ & 11 & 756 & $\begin{array}{c}-7.91(-11.40 \\
-4.43)\end{array}$ & $63 \%$ \\
\hline
\end{tabular}



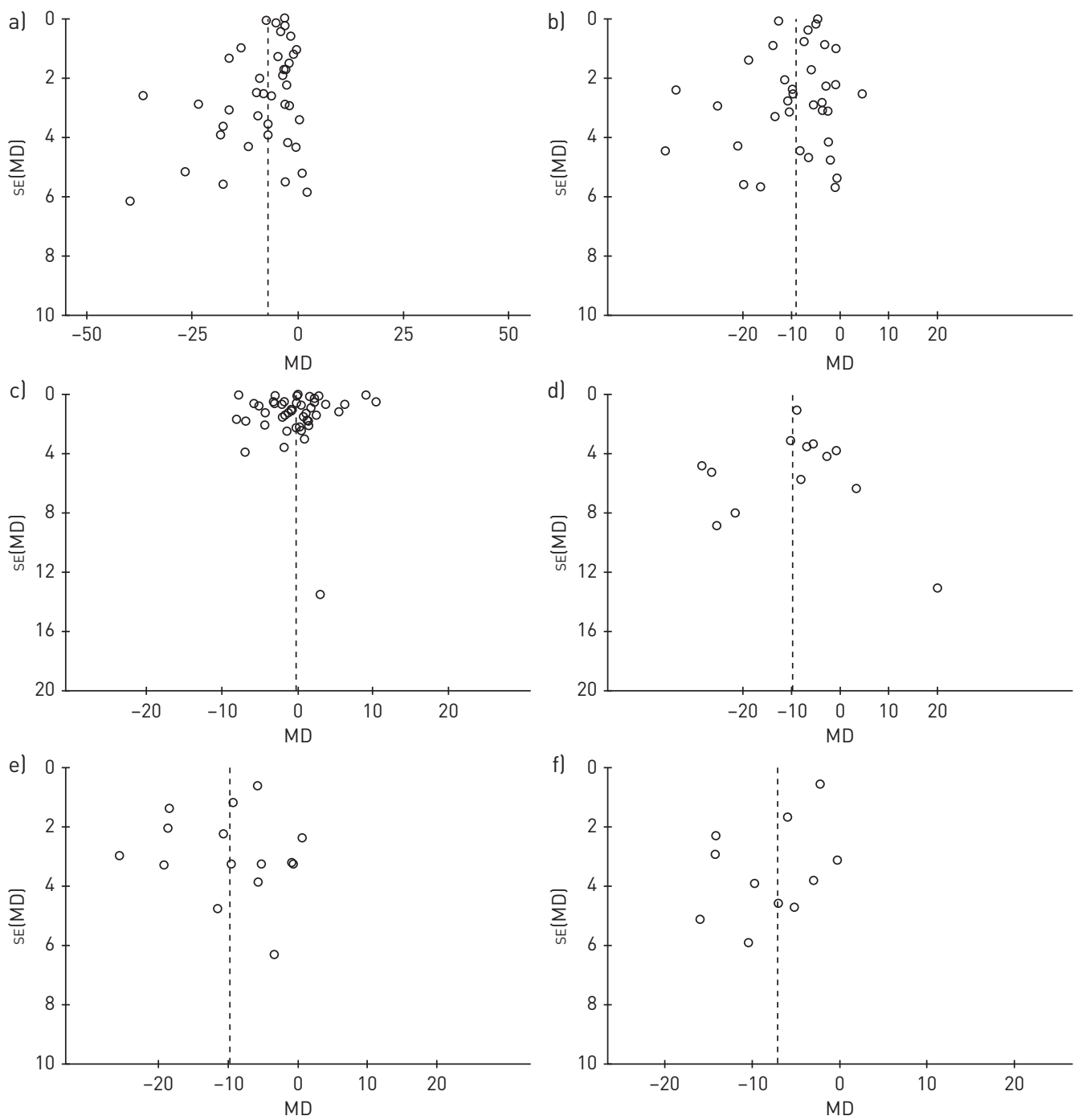

FIGURE 5 Funnel plots of (a) \% predicted forced expiratory volume in $1 \mathrm{~s}$ and (b) \% predicted forced vital capacity, (c) forced expiratory volume in $1 \mathrm{~s} /$ forced vital capacity ratio (\%), (d) forced expiratory flow between $25 \%$ and $75 \%$ of total lung capacity, (e) \% predicted peak expiratory flow, and (f) \% predicted diffusion capacity of the lungs for carbon monoxide.

references S86-S88, S96, S98, S103, S104, S106, S108]. Unlike the studies by VAN DEN BoRST et al. [7] and SAINI et al. [8], our meta-analysis included data on PEF and $\mathrm{FEF}_{25-75 \%}$. The patients with T2D have a reduction of almost $10 \%$ in both of these tests, which indicates that there was impairment both in the large and small airways.

The functional impairment observed in patients with T2D for $\mathrm{FEV}_{1}$ and FVC seem modest but is approximately $300 \mathrm{~mL}$. Much lower differences $(100-150 \mathrm{~mL}$ ) have been considered significant in clinical trials with bronchodilators in patients with COPD [81, 82]. Therefore, pulmonary function impairment in T2D is relevant, although prospective longitudinal studies are still necessary to elucidate the progression of patients with diabetes and pulmonary impairment. It is widely known that patients with T2D have more diseases and pulmonary infections, including pneumonia and tuberculosis [83, 84].

The prevalence of T2D varies according to geographical region and is higher in North America, Southeast Asia and the Middle East [1]. Age, sex, weight, height, body position and ethnicity are factors that affect pulmonary function [85]. We therefore proposed a pre-specified analysis of pulmonary function tests for patients with T2D from various continents. Patients with T2D from all geographical regions presented reduced $\mathrm{FEV}_{1}, \mathrm{FVC}, \mathrm{PEF}, \mathrm{FEF}_{25-75 \%}$ and $D_{\mathrm{LCO}}$. We also found that impairment of T2D in the pulmonary function tests was observed in both sexes and did not change when we included only those studies with nonsmoker patients. In fact, the decrease of pulmonary function tests was higher in studies that included 
only nonsmokers than in the studies with a mixture of smokers and nonsmokers. We do not have an explanation for this finding, but we have observed that most of studies including smokers were conducted in Europe and America. It is possible that patients included in studies from Asia and Africa were nonsmokers but had more environmental exposure to biomass fuel, air pollution or other noxious particles or gases.

Overweight and obesity are associated with a detriment of lung function [86, 87]. Therefore, we could consider that BMI is a confounder. Interestingly, we have observed that the reduction in pulmonary function tests, specifically $\mathrm{FEV}_{1}, \mathrm{FVC}, \mathrm{FEF}_{25-75 \%}$ and $D_{\mathrm{LCO}}$, is present in normal, overweight and obese patients with T2D. FEV 1 reflects the airway resistance, and FVC the total compliance from both the chest wall and the lungs. The fat accumulation on the chest wall and in abdomen substantially alters the movement of thoracic cage and diaphragm and impairs the lung compliance [88].

Including PEF and $\mathrm{FEF}_{25-75 \%}$ is one of novel findings in this meta-analysis. The decrease of \% PEF and \% $\mathrm{FEV}_{1}$ in patients with $\mathrm{T} 2 \mathrm{D}$ was $-9.79 \% \mathrm{y}-7.15 \%$ respectively. It is known that there is high correlation between both parameters. However, while $\mathrm{FEV}_{1}$ is a good indicator of peripheral and proximal airway resistance, PEF reflects the status of proximal airway and is more effort dependant. $\mathrm{FEF}_{25-75 \%}$ is a function of the small airway obstruction. The structural changes of airway and the destruction of the lung parenchyma can modify $\mathrm{FEF}_{25-75 \%}$. Thus, other mechanisms and not only obesity or tobacco use, must be involved in the decrease of lung function in patients with T2D and normal or overweight.

There are structural abnormalities in the lungs of patients with diabetes that could help explain the abnormal pulmonary function test results. Studies on the lungs of obese diabetic rats have observed thickening of alveolar basal lamina [88]. Autopsies of human patients with diabetes have also observed thickening of the capillary and epithelial basement membrane [89, 90]. This thickening is due to inflammatory and fibrotic changes [91]. Fibrosis causes reduced pulmonary elasticity and can decrease lung volumes in T2D. The deterioration of alveolar integrity has also been shown through lung scans following radionuclide inhalation [92]. Alteration of the capillary microcirculation structure can impair pulmonary perfusion and change the ventilation/perfusion ratio [93], which would explain the reduction in $D_{\text {LCO }}$ in patients with T2D.

Various biochemical mechanisms have been proposed to explain the pulmonary damage observed in T2D [94]. Sustained hyperglycaemia causes reduced superoxide dismutase activity and increased oxidative stress. The oxidative stress increases nonenzymatic glycosylation, contributing to pulmonary fibrosis. Abnormalities in the polyol pathways have also been involved, as well as abnormalities in the protein kinase $B$ and nuclear factor- $\kappa \mathrm{B}$ signalling pathways and in transforming growth factor- $\beta[91,95]$.

Heterogeneity is an important finding in our meta-analysis. There are several possible reasons for this. Firstly, there are differences in participants of studies. The mean age of T2D patients ranged from 39.8 to 79 years, the T2D duration from 0.35 to 12.9 years, the mean glycated haemoglobin from 6.1 to $9.5 \%$ and 0-92\% patients had microangiopathy. Even in each continent, there are differences among patients from various geographical regions, for example between Japanese and Iranian in Asia, or Canadian and Venezuelan in America, or German and Greek people in Europe. Secondly, it is possible a publication bias. Probably there are small studies with negative results that have not been published.

One of our study's strengths is the exhaustive literature comprehensive literature search that only excluded Chinese articles. Our additional search provided a large number of articles not collected in the main databases. However, there was a notably high number of articles published in predatory journals, which leads us to think that there are a significant number of studies on pulmonary function in patients with T2D that have not been published, probably due to their low methodological quality. We also performed a sensitivity analysis, observing that the abnormalities in the pulmonary function test results were maintained when we changed statistical analysis method, both with a fixed and a random-effects model. The results also did not change when we differentiated them by study publication date, by including only the good quality studies and even when we excluded the study with the greatest weight, all of which reinforces the results of the meta-analysis.

However, our study also has a number of limitations. Firstly, we resolved the discrepancies in study selection and quality assessment by consensus, and did not calculate the Cohen's $\kappa$. However, the level of interrater agreement was high in study selection and total in quality assessment. Secondly, we observed considerable heterogeneity between the studies, even between those performed in the same geographical region. Although the implementation of a pulmonary function test is standardised, we cannot rule out that the heterogeneity is due to differing methods for measuring the pulmonary parameters. Thirdly, of the 66 studies included in the meta-analysis, only half included 50 or more cases in the T2D group, which leads us to think that many more studies might have been conducted with small groups that have not been 
published. The funnel plots also seem to indicate this idea. However, the results were consistent when we included only the studies with more patients. Finally, only a small number of the studies provided data separated by sex. The results of the analysis by sex should therefore be taken with caution and should be validated in future studies with a large number of patients.

In conclusion, T2D is associated with pulmonary function impairment; however, further studies with large numbers of patients from all geographical areas are needed to corroborate these data and to provide insight into the still pending issues on pulmonary impairment in patients with $\mathrm{T} 2 \mathrm{D}$, specifically progression and possible therapies.

Author contributions: J. Díez-Manglano and U. Asin Samper participated in study design, literature search, data collection, data analysis and data interpretation. J. Díez-Manglano drafted the manuscript, and U. Asin Samper contributed and approved the final version of the manuscript. The corresponding author has full access to all the data in the study and has final responsibility for the decision to submit for publication.

Conflict of interest: J. Díez-Manglano has nothing to disclose. U. Asìn Samper has nothing to disclose.

\section{References}

1 International Diabetes Federation. IDF Diabetes Atlas. 9th Edn, 2019. www.diabetesatlas.org/en/resources/ Date last accessed: May 5, 2020.

2 Organisation for Economic Co-Operation and Development. Health at a glance 2019. OECD indicators. www. oecd-ilibrary.org/social-issues-migration-health/health-at-a-glance-2019_4dd50c09-en Date last accessed: January 22, 2020.

3 Ban CR, Twigg SM. Fibrosis in diabetes complications: pathogenic mechanisms and circulating and urinary markers. Vasc Health Risk Manag 2008; 4: 575-596.

4 Roberts TJ, Burns AT, MacIsaac RJ, et al. Diagnosis and significance of pulmonary microvascular disease in diabetes. Diabetes Care 2018; 41: 854-861.

5 Lange P, Parner J, Schnohr P, et al. Copenhagen City Heart Study: longitudinal analysis of ventilator capacity in diabetic and nondiabetic adults. Eur Respir J 2002; 20: 1406-1412.

6 Kuziemski K, Slominski W, Jassem E. Impact of diabetes mellitus on functional exercise capacity and pulmonary functions in patients with diabetes and healthy persons. BMC Endocr Disord 2019; 19: 2.

7 Van den Borst B, Gosker HR, Zeegers MP, et al. Pulmonary function in diabetes. A metaanalysis. Chest 2010; 138: 393-406.

8 Saini M, Kulandaivelan S, Bansal VK, et al. Pulmonary pathology among patients with type 2 diabetes mellitus: an updated systematic review and meta-analysis. Curr Diabetes Rev 2020; 16: 759-769.

9 Matsubara T, Hara F. The pulmonary function and histopathological studies of the lung in diabetes mellitus. Nippon Ika Daigaku Zashi 1991: 58: 528-536.

10 Lara-Rodríguez DA, Varela González JH, Verlezza S, et al. Disfunción pulmonar en pacientes diabéticos no insulinodependientes. Med Interna (Caracas) 1995; 11: 17-28.

11 Barret-Connor E, Frette C. NIDDM, impaired glucose tolerance, and pulmonary function in older adults. Diabetes Care 1996; 19: 1441-1444.

12 Katoh J, Hara Y, Kurusu M, et al. Cardiorespiratory function as assessed by exercise testing in patients with non-insulin-dependent diabetes mellitus. J Int Med Res 1996; 24: 209-213.

13 Isotani H, Nakamura Y, Kameoka K, et al. Pulmonary diffusing capacity, serum angiotensin-converting enzyme activity and the angiotensin-converting enzyme gene in Japanese non-insulin-dependent diabetes mellitus patients. Diab Res Clin Pract 1999; 43: 173-177.

14 Benbassat CA, Stern E, Kramer M, et al. Pulmonary function in patients with diabetes mellitus. Am J Med Sci 2001; 322: 127-132.

15 Zamarrón C, del Campo F, Paredes C, et al. Estudio de la difusión pulmonar de monóxido de carbono en dos situaciones clínicas: asma bronquial y diabetes mellitus. An Med Intern (Madrid) 2001; 18: 237-242.

16 Ari G, Itil O, Çömlekçi A, et al. Pulmonary function tests and inhalation perfusion scintigraphy findings in patients with type 2 diabetes mellitus. Turk Thorac J 2002; 3: 257-265.

17 Guazzi M, Brambilla R, De Vita S, et al. Diabetes worsens pulmonary diffusion in heart failure, and insulin counteracts this effect. Am J Respir Crit Care Med 2002; 166: 978-982.

18 Maiolo C, Mohamed EI, Di Daniele N, et al. Body composition and pulmonary function in obese type 2 diabetic women. Diab Nutr Metab 2002; 15: 20-25.

19 Boulbou MS, Gourgoulianis K, Petinaki EA, et al. Pulmonary function and circulating adhesion molecules in patients with diabetes mellitus. Can Respir J 2003; 10: 259-264.

20 Guvener N, Tutuncu NB, Ackay S, et al. Alveolar gas exchange in patients with type-2 diabetes mellitus. Endocrine J 2033; 50: 663-667.

21 Melo E, Vianna EO, Gallo L Jr, et al. Pulmonary function, cholinergic bronchomotor tone, and cardiac autonomic abnormalities in type 2 diabetic patients. Braz J Med Biol Res 2003; 36: 291-299.

22 Lau AC, Lo MK, Leung GT, et al. Altered exercise gas exchange as related to microalbuminuria in type 2 diabetic patients. Chest 2004; 125: 1292-1298.

23 Sinha S, Guleria R, Misra A, et al. Pulmonary functions in patients with type 2 diabetes mellitus and correlation with microvascular complications. Indian J Med Res 2004; 119: 66-71.

24 Weisbrod CJ, Eastwwod PR, O’Driscoll GO, et al. Abnormal ventilatory responses to hypoxia in type 2 diabetes. Diabetic Med 2005; 22: 563-568.

25 Meo SA, Al-Drees AM, Arif M, et al. Lung function in type 2 Saudi diabetic patients. Saudi Med J 2006; 27 $338-343$. 
2015; 64: 219-223.

60 Buchmann N, Norman K, Steinhagen-Thiessen E, et al. Lungenfunktion bei älteren Probanden mit metabolischem Syndrom und Typ-2-Diabetes. Ergebnisse der Berliner Altersstudie II. Z Gerontol Geriat 2016; 49: 405-415. 61 Kaur S, Agarwal N. Pulmonary function tests in type 2 diabetes mellitus. Arch Med Health Sci 2016 ; 4: 35-39.

Ortiz-Aguirre AR, Vargas MH, Torres-Cruz A, et al. Cambios espirométricos relacionados con la edad en pacientes diabéticos. Rev Invetig Clin 2006; 58: 109-118.

Özsahin K, Tugrul A, Mert S, et al. Evaluation of pulmonary alveolo-capillary permeability in type 2 diabetes mellitus using technetium 99mTc-DTPA aerosol scintigraphy and carbon monoxide diffusion capacity. J Diab Complications 2006; 20: 205-209.

systemic microangiopathy. Diabetes Care 2008; 31: 1596-1601. Med Colomb 2008; 33: 105-110.

in type 2 diabetes. Diabetologia 2008; 51: 191-197.

Yeh HC, Punjabi NM, Wang NY, et al. Cross-sectional and prospective study of lung function in adults with type 2 diabetes. Diabetes Care 2008; 31: 741-746.

of the disease. J Bangladesh Soc Physiol 2009; 4: 81-87.

Saler T, Cakmak G, Saglam ZA, et al. The assessment of pulmonary diffusing capacity in diabetes mellitus with regard to microalbuminuria. Intern Med 2009; 48: 1939-1943.

Verma S, Goni M, Kudyar RP. Assessment of pulmonary functions in patients with diabetes mellitus. JK Science 2009; 11: 71-74. and their association with microvascular complications. Indian J Chest Dis Allied Sci 2010; 52: 213-216.

Ali O, Begum S, Begum N, et al. PEFR and $\mathrm{FEF}_{25-75 \%}$ in type 2 diabetes mellitus and their relationships with its duration. J Bangladesh Soc Physiol 2010; 5: 14-19.

Lecube A, Sampol G, Muñoz X, et al. Type 2 diabetes impairs pulmonary function in morbidly obese women: a case-control study. Diabetologia 2010; 53: 1210-1216.

Respir Med 2010: 18-22.

Büyükhatipoglu H, Çelik K, Eren MA, et al. [The association between microalbuminuria and carbon monoxide diffusion capacity in patients with type-II diabetes mellitus]. Duzce Med J 2011; 13: 30-35. diabetes mellitus patients. Üzmir GÛs Hastanesi Dergisi 2011; 25: 101-106.

Dharwadkar AR, Dharwadkar AA, Banu G, et al. Reduction in lung functions in type-2 diabetes in Indian population: correlation with glycemic status. Indian J Physiol Pharmacol 2011; 55: 170-175.

Kim HK, Kim CM, Jung YJ, et al. Association of restrictive ventilatory dysfunction with insulin resistance and type 2 diabetes in Koreans. Exp Clin Endocrinol Diabetes 2011; 19: 47-52.

Klein OL, Meltzer D, Carnethon M, et al. Type II diabetes mellitus is associated with decreased measures of lung function in a clinical setting. Respir Med 2011; 105: 1095-1098.

Klein OL, Jones M, Lee J, et al. Reduced lung diffusion capacity in type 2 diabetes is independent of heart failure. Diabetes Res Clin Pract 2012; 96: e73-e75.

Klein OL, Kalhan R, Williams MV, et al. Lung spirometry parameters and diffusion capacity are decreased in patients with type 2 diabetes. Diabet Med 2012; 29: 212-219. 354-357.

Abd-El-Azeem A, Hamdy G, Amin M, et al. Pulmonary function changes in diabetic lung. Egypt J Chest Dis Tuberc 2013; 62: 513-517.

Akber ZA, Al-Edani NI, Khalid LO. Pulmonary function in type 2 diabetic patients in Basrah. N Iraqi J Med 2013; 9: $76-81$. 182-187.

Anandhalakshmi S, Manikandan S, Ganeshkumar P, et al. Alveolar gas exchange and pulmonary functions in patients with type II diabetes mellitus. J Clin Diagn Res 2013; 7: 1874-1877. Diagn Res 2013; 7: 1606-1608.

15: $19-24$

Disord 2013; 12: 15

Shah SH, Sonawane P, Nahar P, et al. Pulmonary function tests in type 2 diabetes mellitus and their association with glycemic control and duration of the disease. Lung India 2013; 30: 108-112.

Diabetes 2014; 122: 322-326.

Jamatia SNN, Wangkheimayum K, Singh WA, et al. Effect of glycemic status on lung function tests in type 2 diabetes mellitus. J Med Soc 2014; 2: 69-72.

Uz-Zaman S, Banerjee J, Singhamahapatra A, et al. Assessment of lung function by spirometry and diffusion study and effect of glycemic control on pulmonary Function in type 2 diabetes mellitus patients of the eastern India. J Clin Diag Res 2014; 8: BC01-4.

Zineldin MAF, Hasan KAG, Al-Adl AS. Respiratory function in type II diabetes mellitus. Egypt J Chest Dis Tuberc 
62 Kumar A, Bade G, Trivedi A, et al. Postural variation of pulmonary diffusing capacity as a marker of lung microangiopathy in Indian patients with type 2 diabetes mellitus. Indian J Endocrinol Metab 2016; 20: 238-244.

63 Caron J, DuManoir GR, Labrecque L, et al. Impact of type 2 diabetes on cardiorespiratory function and exercise performance. Physiol Rep 2017; 5: e13145.

64 Khafaie MA, Salvi SS, Yajnik CS, et al. Air pollution and respiratory health among diabetic and non-diabetic subjects in Pune, India-results from the Wellcome Trust Genetic Study. Environ Sci Pollt Res 2017; 24 15538-15546.

65 Kim HY, Sohn TS, Seok H, et al. Prevalence and risk factors for reduced pulmonary function in diabetic patients: the Korea National Health and Nutrition Examination Survey. Korean J Intern Med 2017; 32: 682-689.

66 López-Cano C, Lecube A, García-Ramírez M, et al. Serum surfactant protein D as a biomarker for measuring lung involvement in obese patients with type 2 diabetes. J Clin Endocrinol Metab 2017; 102: 4109-4116.

67 Nidhi A, Nayyer PS, Rana V, et al. Changes in pulmonary functions in type 2 diabetes mellitus. Indian J Med Spec 2017; 8: 3-6.

68 Shergill N, Kumar A. A study of pulmonary functions in Punjabi type-2 diabetics and non-diabetics. J Exerc Sci Physiother 2017; 13: 60-64.

69 Tai H, Wang M, Zhao Y, et al. Pulmonary function and retrobulbar hemodynamics in subjects with type 2 diabetes mellitus. Respir Care 2017; 62: 602-614.

70 Wilms B, Ernst B, Thurnheer M, et al. Type 2 diabetes is associated with lower cardiorespiratory fitness independent of pulmonary function in severe obesity. Exp Clin Endocrinol Diabetes 2017; 125: 301-306.

71 Okur I, Taspinar B, Atalay OT, et al. The effects of type 2 diabetes mellitus and its complications on physical and pulmonary functions: A case-control study. Physiother Theory Pract 2018; 36: 916-922.

72 Rohling M, Pesta D, Markgraf DF, et al. Metabolic determinants of impaired pulmonary function in patients with newly diagnosed type 2 diabetes mellitus. Exp Clin Endocrinol Diabetes 2018; 126: 584-589.

73 Tayarami A, Moazamian D, Farsi M, et al. Assessment of spirometric indices in patients with type 2 diabetes in Imam Hussein Hospital, Shahroud, Iran (2016-2017). Int J Health Stud 2018; 4: 17-20.

74 Van Eetvelde BLM, Cambier D, Vanden Wyngaert K, et al. The influence of clinically diagnosed neuropathy on respiratory muscle strength in type 2 diabetes mellitus. J Diab Res 2018; 2018: 8065938.

75 Goldman MD. Lung dysfunction in diabetes. Diabetes Care 2003; 26: 195-198.

76 Kaparianos A, Argyropoulou E, Sampsonas F, et al. Pulmonary complications in diabetes mellitus. Chron Respir Dis 2008; 5: 2101-2108.

77 Tiengo A, Fadini GP, Avogaro A. The metabolic syndrome, diabetes and lung dysfunction. Diab Metab 2008; 34 447-454

78 Klein OL, Krishnan JA, Glick S, et al. Systematic review of the association between lung function and type 2 diabetes mellitus. Diabet Med 2010; 27: 977-987.

79 Pitocco D, Fuso L, Conte EG, et al. The diabetic lung - A new target organ? Rev Diabet Stud 2012; 9: 23-35.

80 Lecube A, Simó R, Pallayoba M, et al. Pulmonary function and sleep breathing: two new targets for type 2 diabetes care. Endocrin Rev 2017; 38: 550-573.

81 Tashkin DP, Celli B, Senn S, et al. UPLIFT study investigators. A 4-year trial of tiotropium in chronic obstructive pulmonary disease. N Engl J Med 2008; 359: 1543-1554.

82 Hanania NA, Feldman G, Zachgo W, et al. The efficacy and safety of the novel long-acting $\beta 2$ agonist vilanterol in patients with COPD: a randomized placebo-controlled trial. Chest 2012; 142: 119-127.

83 Ehrlich SF, Quesenberry CP Jr, Van den Eeden SK, et al. Patients diagnosed with diabetes are at increased risk for asthma, chronic obstructive pulmonary disease, pulmonary fibrosis, and pneumonia but not lung cancer Diabetes Care 2010; 33: 55-60.

84 Al-Rifai RH, Pearson F, Critchley JA, et al. Association between diabetes mellitus and active tuberculosis: A systematic review and meta-analysis. PLoS ONE 2017; 12: e0187967.

85 Talaminos Barroso A, Márquez Martín A, Roa Romero LA, et al. Factors affecting lung function: a review of the literature. Arch Bronconeumol 2018; 54: 327-332.

86 Forno E, Hang YY, Mullen J, et al. Overweight, obesity, and lung function in children and adults - a meta-analysis. J Allergy Clin Immunol Pract 2018; 6: 570-581.

87 Wang S, Sun X, Tsia TH, et al. The effect of body mass index on spirometry tests among adults in Xi'an, China. Medicine 2017; 96: e6596.

88 Peters U, Dixon AE. The effect of obesity on lung function. Expert rev Respir Med 2018; 12: 755-767.

89 Foster DJ, Ravikumar P, Bellotto DJ, et al. Fatty diabetic lung: altered alveolar structure and surfactant protein expression. Am J Physiol Lung Cell Mol Physiol 2010; 298: L392-L403.

90 Vracko R, Thorning D, Huang TW. Basal lamina of alveolar epithelium and capillaries: quantitative changes with aging and in diabetes mellitus. Am Rev Respir Dis 1979; 120: 973-983.

91 Weynand B, Jonckheere A, Frans A, et al. Diabetes mellitus induces a thickening of the pulmonary basal lamina. Respiration 1999; 66: 14-19.

92 Talakatta G, Sarikhani M, Muhamed J, et al. Diabetes induces fibrotic changes in the lung through the activation of TGF- $\beta$ signaling pathways. Scient Rep 2018; 8: 11920.

93 Lin CC, Chang CT, Li TC, et al. Objective evidence of impairment of alveolar integrity in patients with non-insulin dependent diabetes mellitus using radionuclide inhalation lung scan. Lung 2002; 180: 181-186.

94 Kuziemski K, Pienkowska J, Slominski W, et al. Pulmonary capillary permeability and pulmonary microangiopathy in diabetes mellitus. Diabetes Res Clin Pract 2015; 108: e56-e59.

95 Zheng H, Wu J, Jin Z, et al. Potential biochemical mechanisms of lung injury in diabetes. Aging Dis 2017; 8: 7-16. 